\title{
The Asymptotic Behaviors of Solutions to the Perturbed Riemann Problem near the Singular Curve for the Chromatography System*
}

Chun Shen

To cite this article: Chun Shen (2015) The Asymptotic Behaviors of Solutions to the Perturbed Riemann Problem near the Singular Curve for the Chromatography System*, Journal of Nonlinear Mathematical Physics 22:1, 76-101, DOI:

https://doi.org/10.1080/14029251.2015.996442

To link to this article: https://doi.org/10.1080/14029251.2015.996442

Published online: 04 January 2021 


\title{
The Asymptotic Behaviors of Solutions to the Perturbed Riemann Problem near the Singular Curve for the Chromatography System*
}

\author{
Chun Shen \\ School of Mathematics and Statistics Science, Ludong University \\ Yantai, Shandong Province, 264025, P.R.China \\ shenchun3641@sina.com
}

Received 1 June 2014

Accepted 29 September 2014

\begin{abstract}
The Riemann problem for a simplified chromatography system is considered and the global Riemann solutions are constructed in all kinds of situations. In particular, the zero rarefaction wave, the zero shock wave and the zero delta shock wave are discovered in the Riemann solutions in some limit situations, which have infinite propagation speeds. Furthermore, these zero waves are analyzed by introducing the so-called double Riemann problem with three pieces of constant states. More precisely, we take the approximations of zero waves and investigate wave interactions in details before the limits are taken.
\end{abstract}

Keywords: Chromatography system; zero wave; delta shock wave; wave interaction; Riemann problem.

2000 Mathematics Subject Classification: 35L65, 35L67, 76N15

\section{Introduction}

The theory of nonlinear chromatography system is the foundation of chromatography separation process and also plays an important role in the modern industry. In this paper, we consider the Riemann problem for a simplified chromatography system

$$
\left\{\begin{array}{l}
\partial_{t} v+\partial_{x}\left(\frac{v}{1+v}\right)=0 \\
\partial_{t} w+\partial_{x}\left(\frac{w}{1+v}\right)=0
\end{array}\right.
$$

with the initial data

$$
(v, w)(x, 0)= \begin{cases}\left(v_{l}, w_{l}\right), & -\infty<x<0 \\ \left(v_{r}, w_{r}\right), & 0<x<+\infty\end{cases}
$$

where all the $v_{l, r}$ and $w_{l, r}$ are constants.

The system (1.1) can be derived directly from the nonlinear chromatography system which depicts the chromatography separation of two chemical species $\left(u_{1}\right.$ and $\left.u_{2}\right)$ that transform into each

*This work is supported by National Natural Science Foundation of China (11441002,11271176). 
other with first-order kinetic as they pass through the Langmuir isotherm reactor $[3,28,31,40]$

$$
\left\{\begin{array}{l}
\partial_{t} u_{1}+\partial_{x}\left(\frac{u_{1}}{1+u_{1}+u_{2}}\right)=0 \\
\partial_{t} u_{2}+\partial_{x}\left(\frac{u_{2}}{1+u_{1}+u_{2}}\right)=0
\end{array}\right.
$$

by introducing the change of variables

$$
v=u_{1}+u_{2} \quad \text { and } \quad w=u_{1}-u_{2}
$$

Here both $u_{1}$ and $u_{2}$ are the non-negative functions of the variables $(x, t) \in R \times R_{+}$, which denote transformations of the concentrations of two chemical species.

The system (1.1) is the simplest example for systems of Temple class [40]. In other words, the shock curves coincide with the rarefaction curves in the phase plane. Compared with general systems of conservation laws, well-posed results for systems of Temple class [1] are available for a much larger class of initial data due to the above feature. Furthermore, thanks to the particular structure, wave interactions for the systems of Temple class can also be dealt with completely.

Recently, Ambrosio et al [1] have rewritten the system (1.3) into the system (1.1) by introducing the change of variables (1.4). Then, they have studied the system (1.1) as an example by employing new well-posedness results for continuity and transport equations. Their method is to split the chromatography system in the coupling between a scalar conservation law and a transport equation such that the transport equation techniques can be applied. Bressan and Shen [4] have introduced a different change of variables $v=u_{1}+u_{2}$ and $\theta=u_{2} / u_{1}$ for the system (1.3) where they were mainly concerned with the study of ODEs with discontinuous vector fields.

On the other hand, the system (1.1) can also be derived from the other nonlinear chromatography system with the generalized Langmuir isotherm reactor $[6,21,22,41]$

$$
\left\{\begin{array}{l}
\partial_{t} u_{1}+\partial_{x}\left(\frac{u_{1}}{1-u_{1}+u_{2}}\right)=0 \\
\partial_{t} u_{2}+\partial_{x}\left(\frac{u_{2}}{1-u_{1}+u_{2}}\right)=0
\end{array}\right.
$$

by introducing the change of variables

$$
v=u_{2}-u_{1} \quad \text { and } \quad w=u_{1}+u_{2}
$$

Here $u_{1}$ and $u_{2}$ are also the non-negative functions of the variables $(x, t) \in R \times R_{+}$to denote the Langmuir-like and the anti Langmuir-like components respectively which express transformations of the concentrations of two chemical species. Moreover, $1-u_{1}+u_{2}>0$ should be satisfied.

The substantial difference between the new model (1.5) and the model (1.3) lies in that the flux functions $\frac{u_{i}}{1-u_{1}+u_{2}}(i=1,2)$ in (1.5) take the place of $\frac{u_{i}}{1+u_{1}+u_{2}}(i=1,2)$ in (1.3). In the chromatography theory, the new model (1.5) can be explained as the interaction of a competitivecooperative relation between two species. In contrast, the model (1.3) can be explained as the interaction of a competitive-competitive relation between two species. If we adopt the generalized Langmuir isotherm reactor, then the delta measure may appear in the two chemical species $u_{1}$ and $u_{2}$.

Recently, the delta shock wave has been captured numerically and experimentally by Mazzotti et al $[21,22]$ in the Riemann solutions for the local equilibrium model of two-component nonlinear 
chromatography which consists of the following system of conservation laws

$$
\left\{\begin{array}{l}
\partial_{t}\left(u_{1}+\frac{a_{1} u_{1}}{1-u_{1}+u_{2}}\right)+\partial_{x} u_{1}=0, \\
\partial_{t}\left(u_{2}+\frac{a_{2} u_{2}}{1-u_{1}+u_{2}}\right)+\partial_{x} u_{2}=0
\end{array}\right.
$$

with some specified Riemann initial data, in which $u_{1}$ and $u_{2}$ are the concentrations of the two absorbing species which should satisfy $u_{1}, u_{2} \geq 0,1-u_{1}+u_{2}>0$ and $a_{2}>a_{1}>0$. Since then, extensive studies have been carried out on the formation of delta shock wave solution to all kinds of chromatography systems $[6,13,41]$.

In the present paper, we are mainly interested in the simplified chromatography system (1.1) due to the fact that (1.1) reflects the most fundamental properties of chromatography system. Sun [37] discovered that a delta shock wave solution appears in the situation $v_{l}=0<v_{r}$ for the Riemann problem (1.1) and (1.2) by employing the self-similiar viscosity vanishing approach. Furthermore, $\mathrm{Li}$ and Shen [20] has generalized the result in [37] to the general situation $-1<v_{l} \leq 0<v_{r}$ recently. If the system (1.1) comes from (1.5) to describe the interaction of a competitive-cooperative relation between two species, then it is natural to consider the system (1.1) in the phase space $(v, w) \in$ $(-1, \infty) \times[0, \infty)$. Here we notice that the delta measure appears in $w$ for the Riemann problem (1.1) and (1.2) provided that the Riemann initial data satisfy $-1<v_{l} \leq 0<v_{r}$. In other words, the delta measures appear in both the state variables $u_{1}$ and $u_{2}$ for the system (1.5).

For the system (1.1), the line $v=0$ in the phase space $(v, w)$ plays a very important role since both the two eigenvalues become 1 when $v=0$. Thus, we can see that the system (1.1) possesses a parabolic degeneracy on the line $v=0$ in the phase space $(v, w)$. Compared with the results in $[32,38]$, wave interaction for the system $(1.1)$ in the phase space $(v, w) \in(-1, \infty) \times[0, \infty)$ has a more complicated structure due to the fact that a parabolic degeneracy appears although the system (1.1) belongs to Temple class.

For the Riemann problem (1.1) and (1.2) in the situation $-1<v_{r}<v_{l}$, it is interesting to notice that the propagation speed of the wave front of rarefaction wave for the second family tends to $+\infty$ if we take the limit $v_{r} \rightarrow(-1)^{+}$. This is to say, the wave front of rarefaction wave is consistent with the positive $x$-axis in the limit $v_{r} \rightarrow(-1)^{+}$situation which is called as the zero rarefaction wave for the sake of convenience. On the other hand, for the Riemann problem (1.1) and (1.2) in the situation $-1<v_{l}<v_{r}<0$, we can also see that both the propagation speeds of the contact discontinuity and shock wave tend to $+\infty$ if we take $v_{l} \rightarrow(-1)^{+}$. In other words, both of them coincide with the positive $x$-axis, which is also called as the zero shock wave. Finally, in the situation $-1<v_{l} \leq 0<v_{r}$, the propagation speed of the delta shock wave also tends to $+\infty$ in the limit $v_{l} \rightarrow(-1)^{+}$situation in the Riemann solution to (1.1) and (1.2). So, the delta shock wave in this limit situation is also called as the zero delta shock wave. In addition, the strength of the delta shock wave also tends to $+\infty$ for any given time $t>0$ in the limit $v_{l} \rightarrow(-1)^{+}$situation.

Compared with hyperbolic systems of conservation laws to describe gas dynamics, the roles for the variables $x$ and $t$ in the above chromatography systems are opposite. More precisely, $x$ is related to the time and $t$ denotes the location down the reactor. Thus the above zero waves to the simplified chromatography system (1.1) is equivalent to the standing wave for the gas dynamics in the physics sense. In fact, the zero waves play the critical roles in analyzing the chromatography systems for the reason that it can be used to depict the extreme situation without the exchange kinetics between the two chemical species $[21,22,28,31]$. 
It is clear to see that the Riemann solution to (1.1) and (1.2) becomes sensitive to the choice of initial data if $v_{l}$ or $v_{r}$ approaches the singular value -1 . Since the speeds of these zero waves become infinite such that information can be exchanged instantaneously between neighboring Riemann problems, which implies that there is no information to exchange between two species in the real chromatography system. On the other hand, it is noticed that the Riemann problem (1.1) and (1.2) cannot describe the dynamical picture for the system (1.1) in all situations. In other words, the Riemann solution to (1.1) and (1.2) cannot be considered separately when a zero wave appears.

Motivated by the above requirements, we are interested in the behavior when the state is near the singular curve $v=-1$ in the phase space. In order to investigate it in a fully explicit form, we should consider the so-called double Riemann problem with three pieces of constant states such as (3.1) below for the system (1.1). We take the zero shock wave for example to explain it, which appears if we take the limit $v_{l} \rightarrow(-1)^{+}$in the solution to (1.1) and (3.1) when $v_{l}<v_{m}<0$. In order to deal with it, we need to take $-1<v_{l}<-1+\varepsilon$ for sufficiently small $\varepsilon>0$ as an approximation of the value -1 to mediate an instantaneous interaction and then investigate the approximate double Riemann problem by employing the method of characteristics. Finally, we take the limit $v_{l} \rightarrow(-1)^{+}$ in the solution to the approximate double Riemann problem in order to analyze the dynamic of zero wave.

Actually, the zero rarefaction wave $[2,42]$ and the zero shock wave [11] which appears usually when the flux function is discontinuous, are also discovered in the Riemann solutions in some limit situations. The zero waves were discovered and proposed initially by Gimse [11] when he studied the Riemann problem for a scalar conservation law

$$
\partial_{t} u+\partial_{x} f(u)=0
$$

in which the flux function $f$ is assumed to be piecewise smooth with a finite number of jump discontinuities, for example a simplest situation that $f$ has only one jump discontinuous point $u_{0}$ such that $\lim _{u \rightarrow u_{0}^{-}} f(u) \neq \lim _{u \rightarrow u_{0}^{+}} f(u)$. In [11], the zero shock waves were identified which are discontinuous in the solutions that carry no information and propagate with infinite speed. In [10], Dias and Figueira used the technique of smooth mollifier to smooth out discontinuities in the flux function locally. Moreover, they proved that the solutions of the mollified problem converge to those of the original problem in the limit situation. Recently, the zero waves have also been used to depict the phenomenon of traffic flow [42] and production flows and supply chains with finite buffers $[2,12]$.

About the measure-valued solutions for hyperbolic systems of conservation laws, such as the delta shock wave and the singular shock wave, there are numerous excellent papers for the related equations and results, see $[5,7,15,18,29,33,36,39,43]$ for instance. There are also several methods to deal with the interaction problem about the delta shock wave, such as by using the generalized Rankine-Hugoniot condition proposed by $\mathrm{Li}$ et al. [19] and used in [30,41], the weak asymptotic method proposed by Danilov and Shelkovich $[8,9]$ and the method of splitting of delta function proposed by Nedeljkov [23,24] and used in [14,27,34,35,38]. Recently, the new concept of shadow wave proposed by Nedeljkov $[25,26]$ can also be well used to study the interaction problem about the delta shock wave.

The paper is organized as follows. In section 2, the Riemann problem for (1.1) and (1.2) is considered when $(v, w) \in(-1, \infty) \times[0, \infty)$. Then, the zero waves including zero shock wave, zero delta shock wave and zero rarefaction wave are obtained in the limit situation. In section 3 , in order to analyze the zero shock wave, we consider the so-called double Riemann problem (1.1) and (3.1) 
by requiring $-1<v_{l}<v_{m}<0$ and then taking the limit $v_{l} \rightarrow(-1)^{+}$. In section 4 , we also consider (1.1) and (3.1) by requiring $-1<v_{l}<0<v_{m}$ and then taking the limit $v_{l} \rightarrow(-1)^{+}$to study the zero delta shock wave, in which the strength of the delta shock wave is calculated completely during the process of penetration. In section 5 , in order to study the zero rarefaction wave, we take $v_{m}$ near -1 and also deal with the so-called double Riemann problem (1.1) and (3.1) before the limit $v_{m} \rightarrow(-1)^{+}$is taken.

\section{Zero Waves in the Limit of Riemann Solutions to (1.1) and (1.2)}

In this section, we consider the Riemann problem for (1.1) and (1.2) in the phase space $(v, w) \in$ $(-1, \infty) \times[0, \infty)$. In what follows, we first depict the properties of $(1.1)$ and then list the Riemann solutions to (1.1) and (1.2) case by case. We can refer to [6, 20,32, 37, 38, 41] for the Riemann problem about the nonlinear chromatography system.

The eigenvalues of (1.1) are $\lambda_{1}=\frac{1}{(1+v)^{2}}$ and $\lambda_{2}=\frac{1}{1+v}$ respectively. Thus, it is clear that (1.1) is strictly hyperbolic in the phase space $-1<v<0$ and $v>0$ for $\lambda_{1} \neq \lambda_{2}$ and is non-strictly hyperbolic on the line $v=0$ for $\lambda_{1}=\lambda_{2}$. The corresponding right eigenvectors are $\overrightarrow{r_{1}}=\left(\frac{v}{\sqrt{v^{2}+w^{2}}}, \frac{w}{\sqrt{v^{2}+w^{2}}}\right)^{T}$ and $\overrightarrow{r_{2}}=(0,1)^{T}$ respectively. Thus $\lambda_{1}$ is genuinely nonlinear provided that $v \neq 0$ and $\lambda_{2}$ is always linearly degenerate. Therefore, the associated waves are rarefaction waves $R$ or shock waves $S$ for the first family and contact discontinuities $J$ for the second family. The Riemann invariants along the characteristic fields are $\frac{w}{v}$ and $v$ respectively. Let us denote $\xi=\frac{x}{t}$ for conciseness, then we are in a position to deliver the Riemann solutions to (1.1) and (1.2) according to the value of $v$ as follows:

(1) If $v_{l}>v_{r}>0$, then the Riemann solution of (1.1) and (1.2) is $R+J$ which can be expressed as:

$$
(v, w)(x, t)= \begin{cases}\left(v_{l}, w_{l}\right), & \xi<\lambda_{1}\left(v_{l}\right), \\ R, & \lambda_{1}\left(v_{l}\right) \leq \xi \leq \lambda_{1}\left(v_{r}\right), \\ \left(v_{r}, \frac{v_{r} w_{l}}{v_{l}}\right), & \lambda_{1}\left(v_{r}\right)<\xi<\tau \\ \left(v_{r}, w_{r}\right), & \xi>\tau .\end{cases}
$$

in which the state $(v, w)$ in $R$ is determined by $\frac{w}{v}=\frac{w_{l}}{v_{l}}$ and the propagation speed of $J$ is $\tau=\frac{1}{1+v_{r}}$.

In particular, if $v_{r}=0$, then we can acquire the relation $\lambda_{1}\left(v_{r}\right)=\tau=1$. In other words, a composite wave $R J$ is formed when $v_{r}=0$ due to the fact that the wave front of $R$ is in accordance with $J$.

(2) If $0<v_{l}<v_{r}$, then the Riemann solution of (1.1) and (1.2) is $S+J$ in the form:

$$
(v, w)(x, t)= \begin{cases}\left(v_{l}, w_{l}\right), & \xi<\sigma \\ \left(v_{r}, \frac{v_{r} w_{l}}{v_{l}}\right), & \sigma<\xi<\tau, \\ \left(v_{r}, w_{r}\right), & \xi>\tau\end{cases}
$$

in which the propagation speeds of $S$ and $J$ are $\sigma=\frac{1}{\left(1+v_{l}\right)\left(1+v_{r}\right)}$ and $\tau=\frac{1}{1+v_{r}}$ where $0<\sigma<$ $\tau<1$.

Similarly, if $v_{r}=0$, then we also have the relation $\sigma=\tau=1$. In other words, a composite wave $S J$ is formed when $v_{r}=0$ since the line of $S$ is identical with that of $J$. 
(3) If $-1<v_{r}<0<v_{l}$, then the Riemann solution of (1.1) and (1.2) is $R_{1}+R_{2}$ which is given by

$$
(v, w)(x, t)= \begin{cases}\left(v_{l}, w_{l}\right), & \xi<\lambda_{1}\left(v_{l}\right), \\ R_{1}, & \lambda_{1}\left(v_{l}\right) \leq \xi<1, \\ R_{2}, & 1<\xi \leq \lambda_{1}\left(v_{r}\right), \\ \left(v_{r}, w_{r}\right), & \xi>\lambda_{1}\left(v_{r}\right) .\end{cases}
$$

It should be emphasized that the state $(v, w)$ in $R_{1}$ is determined by $\frac{w}{v}=\frac{w_{l}}{v_{l}}$, otherwise the state $(v, w)$ in $R_{2}$ is determined by $\frac{w}{v}=\frac{w_{r}}{v_{r}}$. The line $x=t$ can be seen as a contact discontinuity $J$ to separate $R_{1}$ and $R_{2}$.

If we take the limit $v_{r} \rightarrow(-1)^{+}$in (2.3), then we have

$$
\lim _{v_{r} \rightarrow(-1)^{+}} \lambda_{1}\left(v_{r}\right)=\lim _{v_{r} \rightarrow(-1)^{+}} \frac{1}{\left(1+v_{r}\right)^{2}}=+\infty,
$$

which implies that the propagation speed of the wave front of $R_{2}$ tends to $+\infty$. In other words, the wave front of $R_{2}$ is consistent with the positive $x$-axis, which is called as the zero rarefaction wave in $[2,42]$ which appears usually when the flux function is discontinuous.

(4) If $-1<v_{l}<v_{r}<0$, then the Riemann solution of (1.1) and (1.2) is $J+S$ as follows:

$$
(v, w)(x, t)= \begin{cases}\left(v_{l}, w_{l}\right), & \xi<\tau, \\ \left(v_{l}, \frac{v_{l} w_{r}}{v_{r}}\right), & \tau<\xi<\sigma, \\ \left(v_{r}, w_{r}\right), & \xi>\sigma,\end{cases}
$$

in which the propagation speed of $J$ is $\tau=\frac{1}{1+v_{l}}$ and that of $S$ is $\sigma=\frac{1}{\left(1+v_{l}\right)\left(1+v_{r}\right)}$. Here we notice that $1<\tau<\sigma$.

Similarly, if we take the limit $v_{l} \rightarrow(-1)^{+}$in (2.5), then we also have

$$
\begin{gathered}
\lim _{v_{l} \rightarrow(-1)^{+}} \tau=\lim _{v_{l} \rightarrow(-1)^{+}} \frac{1}{1+v_{l}}=+\infty, \\
\lim _{v_{l} \rightarrow(-1)^{+}} \sigma=\lim _{v_{l} \rightarrow(-1)^{+}} \frac{1}{\left(1+v_{l}\right)\left(1+v_{r}\right)}=+\infty,
\end{gathered}
$$

which means that both the propagation speeds of $J$ and $S$ tend to $+\infty$. In other words, both $J$ and $S$ coincide with the positive $x$-axis and the middle fan region $\tau<\xi<\sigma$ with the immediate state $\left(v_{l}, \frac{v_{l} w_{r}}{v_{r}}\right)$ disappears, which is called as the zero shock wave in [11] which also appears when the flux function is discontinuous.

(5) If $-1<v_{r}<v_{l}<0$, then the Riemann solution of (1.1) and (1.2) is $J+R$ as follows:

$$
(v, w)(x, t)= \begin{cases}\left(v_{l}, w_{l}\right), & x<\tau t \\ \left(v_{l}, \frac{v_{l} w_{r}}{v_{r}}\right), & \tau<\xi<\lambda_{1}\left(v_{l}\right), \\ R, & \lambda_{1}\left(v_{l}\right) \leq \xi \leq \lambda_{1}\left(v_{r}\right), \\ \left(v_{r}, w_{r}\right), & \xi>\lambda_{1}\left(v_{r}\right),\end{cases}
$$


in which the propagation speed of $J$ is $\tau=\frac{1}{1+v_{l}}>1$ and the state $(v, w)$ in $R$ is determined by $\frac{w}{v}=\frac{w_{r}}{v_{r}}$. If we take the limit $v_{r} \rightarrow(-1)^{+}$in (2.8), then the limit situation is similar to that in Case 3. In other words, the zero rarefaction wave also appears in the limit $v_{r} \rightarrow(-1)^{+}$situation.

(6) If $-1<v_{l}<0<v_{r}$, from the exact definition of a delta shock wave solution in the framework introduced in $[8,9]$ and used in $[16,17,41]$, then one can see that the Riemann problem (1.1) and (1.2) owns a piecewise smooth solution which is a delta shock wave connecting the two constants $\left(v_{l}, w_{l}\right)$ and $\left(v_{r}, w_{r}\right)$ in the form:

$$
(v, w)(x, t)=\left\{\begin{array}{l}
\left(v_{l}, w_{l}\right), \quad \xi<\sigma_{\delta} \\
\left(v_{\delta}, \beta(t) \delta\left(x-\sigma_{\delta} t\right)\right), \quad \xi=\sigma_{\delta} \\
\left(v_{r}, w_{r}\right), \quad \xi>\sigma_{\delta}
\end{array}\right.
$$

where

$$
v_{\delta}=v_{l}+v_{r}+v_{l} v_{r}, \quad \sigma_{\delta}=\frac{1}{1+v_{\delta}}=\frac{1}{\left(1+v_{l}\right)\left(1+v_{r}\right)}, \quad \beta(t)=\frac{\left(v_{r} w_{l}-v_{l} w_{r}\right) t}{\left(1+v_{l}\right)\left(1+v_{r}\right)}
$$

Let us check briefly that the measure solution (2.9) with (2.10) should satisfy the generalized Rankine-Hugoniot condition as follows:

$$
\left\{\begin{array}{l}
\frac{d x}{d t}=\sigma_{\delta} \\
\frac{d \beta(t)}{d t}=\sigma_{\delta}[w]-\left[\frac{w}{1+v}\right] \\
\sigma_{\delta}[v]=\left[\frac{v}{1+v}\right]
\end{array}\right.
$$

In fact, if $-1<v_{l}<0<v_{r}$, then we assume that the position of delta shock wave is $x=x(t)$ and the values of $v$ and $w$ on this delta shock wave curve are $v_{\delta}$ and $\beta(t) \delta(x-x(t))$ respectively. Let us assume $x=x(t)>0$ for all the time $t$ for simplicity. Then, for any test function $\psi(x, t) \in C_{c}^{\infty}\left(R \times R_{+}\right)$, we have

$$
\begin{aligned}
I & =\int_{0}^{\infty} \int_{-\infty}^{\infty}\left(v \psi_{t}+\frac{v}{1+v} \psi_{x}\right) d x d t \\
& =\int_{0}^{\infty} \int_{-\infty}^{x(t)}\left(v_{l} \psi_{t}+\frac{v_{l}}{1+v_{l}} \psi_{x}\right) d x d t+\int_{0}^{\infty} \int_{x(t)}^{\infty}\left(v_{r} \psi_{t}+\frac{v_{r}}{1+v_{r}} \psi_{x}\right) d x d t \\
& =\int_{0}^{\infty} \int_{t(x)}^{\infty} v_{l} \psi_{t} d t d x+\int_{0}^{\infty} \int_{0}^{t(x)} v_{r} \psi_{t} d t d x+\int_{0}^{\infty}\left(\frac{v_{l}}{1+v_{l}}-\frac{v_{r}}{1+v_{r}}\right) \psi(x(t), t) d t \\
& =\int_{0}^{\infty}\left(v_{r}-v_{l}\right) \psi(x, t(x)) d x+\int_{0}^{\infty}\left(\frac{v_{l}}{1+v_{l}}-\frac{v_{r}}{1+v_{r}}\right) \psi(x(t), t) d t
\end{aligned}
$$

in which $t=t(x)$ is the inverse function of $x=x(t)$ and the fact that $\psi$ is compact support in $R_{+}^{2}$ has been used. Thus, the propagation speed of the delta shock wave $\sigma_{\delta}=\frac{d x}{d t}$ should satisfy $\sigma_{\delta}[v]=\left[\frac{v}{1+v}\right]$ 
for $I$ vanishes for any test function $\psi(x, t) \in C_{c}^{\infty}\left(R \times R_{+}\right)$. Analogously, we also have

$$
\begin{aligned}
I I= & \int_{0}^{\infty} \int_{-\infty}^{\infty}\left(w \psi_{t}+\frac{w}{1+v} \psi_{x}\right) d x d t \\
= & \int_{0}^{\infty} \int_{-\infty}^{x(t)}\left(w_{l} \psi_{t}+\frac{w_{l}}{1+v_{l}} \psi_{x}\right) d x d t+\int_{0}^{\infty} \int_{x(t)}^{\infty}\left(w_{r} \psi_{t}+\frac{w_{r}}{1+v_{r}} \psi_{x}\right) d x d t \\
& +\int_{0}^{\infty} \beta(t)\left(\psi_{t}(x(t), t)+\frac{1}{1+v_{\delta}} \psi_{x}(x(t), t)\right) d t \\
= & \int_{0}^{\infty}\left(w_{r}-w_{l}\right) \psi(x, t(x)) d x+\int_{0}^{\infty}\left(\frac{w_{l}}{1+v_{l}}-\frac{w_{r}}{1+v_{r}}\right) \psi(x(t), t) d t+\int_{0}^{\infty} \beta(t) d \psi(x(t), t) .
\end{aligned}
$$

Thus, one can see that the second equality in (2.11) should be satisfied for the same reason that $I I$ vanishes for any test function $\psi(x, t) \in C_{c}^{\infty}\left(R \times R_{+}\right)$.

In order to ensure the uniqueness of the delta shock wave solution to the Riemann problem (1.1) and (1.2) when $-1<v_{l}<0<v_{r}$, the over-compressive entropy condition should be proposed. It is worthwhile to notice that $\lambda_{1}<\lambda_{2}$ for $v>0$ and $\lambda_{1}>\lambda_{2}$ for $-1<v<0$ here. Thus, if $-1<v_{l}<$ $0<v_{r}$, then the over-compressive entropy condition for delta shock wave

$$
\lambda_{1}\left(v_{r}, w_{r}\right)<\lambda_{2}\left(v_{r}, w_{r}\right)<\sigma_{\delta}<\lambda_{2}\left(v_{l}, w_{l}\right)<\lambda_{1}\left(v_{l}, w_{l}\right)
$$

namely

$$
\frac{1}{\left(1+v_{r}\right)^{2}}<\frac{1}{1+v_{r}}<\sigma_{\delta}<\frac{1}{1+v_{l}}<\frac{1}{\left(1+v_{l}\right)^{2}}
$$

should be proposed, which means that all the characteristics enter the delta shock wave curve from both sides.

From the above discussion, we can see that if we restrict ourselves in the phase space $(v, w) \in$ $(-1, \infty) \times[0, \infty)$, then the generalized Rankine-Hugoniot condition and the over-compressive entropy condition can be used to the construction of delta shock wave solution to the Riemann problem (1.1) and (1.2) when $-1<v_{l}<0<v_{r}$. In fact, if we want to extend our result in the phase space $(v, w) \in(-\infty, \infty) \times[0, \infty)$, then the technique developed by Kalisch and Mitrovic [16] may be used to attack the existence and uniqueness of the delta shock wave solution in some certain situations. This problem is left for our future work.

If we take the limit $v_{l} \rightarrow(-1)^{+}$in the Riemann solution (2.9) with (2.10), then we have

$$
\begin{aligned}
\lim _{v_{l} \rightarrow(-1)^{+}} v_{\delta} & =\lim _{v_{l} \rightarrow(-1)^{+}}\left(v_{l}+v_{r}+v_{l} v_{r}\right)=-1, \\
\lim _{v_{l} \rightarrow(-1)^{+}} \sigma_{\delta} & =\lim _{v_{l} \rightarrow(-1)^{+}} \frac{1}{\left(1+v_{l}\right)\left(1+v_{r}\right)}=+\infty,
\end{aligned}
$$

and

$$
\lim _{v_{l} \rightarrow(-1)^{+}} \beta(t)=\lim _{v_{l} \rightarrow(-1)^{+}} \frac{\left(v_{r} w_{l}-v_{l} w_{r}\right) t}{\left(1+v_{l}\right)\left(1+v_{r}\right)}=+\infty
$$

for any given time $t>0$.

Then, the propagation speed of the delta shock wave tends to $+\infty$ in the limit $v_{l} \rightarrow(-1)^{+}$ situation. In addition, the strength of the delta shock wave tends to $+\infty$ for any given time $t>0$ in 
the limit $v_{l} \rightarrow(-1)^{+}$situation. So, the delta shock wave in this limit situation can also be called as the zero delta shock wave.

\section{Analysis of Zero Shock Wave}

With the Riemann solutions to (1.1) and (1.2) in hand, it is natural to consider the so-called double Riemann problem since the Riemann problem (1.1) and (1.2) cannot describe the dynamic pictures in all situations. From now on, we are interested in the following special initial data with three pieces of constant states

$$
(v, w)(x, 0)= \begin{cases}\left(v_{l}, w_{l}\right), & -\infty<x<0 \\ \left(v_{m}, w_{m}\right), & 0<x<1 \\ \left(v_{r}, w_{r}\right), & 1<x<+\infty .\end{cases}
$$

In this section, we are concerned with the situation that the zero shock wave interacts with the other elementary waves. In order to deal with it, we take $-1<v_{l}<-1+\varepsilon$ for sufficiently small $\varepsilon>0$ and $v_{l}<v_{m}<0$, and then we pass to the limit $v_{l} \rightarrow(-1)^{+}$. Thus, in the limit $v_{l} \rightarrow(-1)^{+}$ situation, the Riemann solution to (1.1) and (1.2) at the point $(0,0)$ is a zero shock wave. In other words, we take the approximation of the zero shock wave in the Riemann solution at the point $(0,0)$. One can see that all the waves on the right become zero shock waves instantaneously in the limit $v_{l} \rightarrow(-1)^{+}$situation.

Remark 3.1. We know that $x$ in the system (1.1) denotes the time in the sense of chromatography theory. Thus, the Riemann initial data (1.2) and the initial data (3.1) do not make sense. In fact, we should use the following initial boundary condition

$$
(v, w)(0, t)=\left(v_{l}, w_{l}\right) \text { for } t>0 \text { and }(v, w)(x, 0)=\left(v_{r}, w_{r}\right) \text {, for } x>0
$$

instead of the Riemann initial data (1.2) and also use

$$
(v, w)(0, t)=\left(v_{l}, w_{l}\right) \text { for } t>0 \quad \text { and } \quad(v, w)(x, 0)= \begin{cases}\left(v_{m}, w_{m}\right), & 0<x<1 \\ \left(v_{r}, w_{r}\right), & 1<x<+\infty\end{cases}
$$

instead of the initial data (3.1). We notice that all the propagation speeds of waves are positive in the Riemann solutions to (1.1) and (1.2) in Section 2. Thus the initial boundary problem (1.1) and (3.3) can be dealt with similarly. Here we consider the so-called double Riemann problem (1.1) and (3.1) in the habit of hyperbolic systems of conservation laws.

Case 3.1. $-1<v_{l}<v_{m}<v_{r}<0$

In this case, we consider that $J+\vec{S}$ emits from $(1,0)$ which happens when $-1<v_{l}<v_{m}<$ $v_{r}<0$ is satisfied (see Fig.3.1). For sufficiently small $t$, the solution of the Cauchy problem (1.1) and (3.1) can be expressed by the juxtaposition of the solutions to the Riemann problem for (1.1) at the points $(0,0)$ and $(1,0)$. In other words, when $t$ is small enough, the solution of the Cauchy problem (1.1) and (3.1) can be given briefly as (see Fig.1):

$$
\left(v_{l}, w_{l}\right)+J_{1}+\left(v_{1}, w_{1}\right)+S_{1}+\left(v_{m}, w_{m}\right)+J_{2}+\left(v_{2}, w_{2}\right)+S_{2}+\left(v_{r}, w_{r}\right),
$$

in which

$$
\left(v_{1}, w_{1}\right)=\left(v_{l}, \frac{v_{l} w_{m}}{v_{m}}\right) \quad \text { and } \quad\left(v_{2}, w_{2}\right)=\left(v_{m}, \frac{v_{m} w_{r}}{v_{r}}\right)
$$




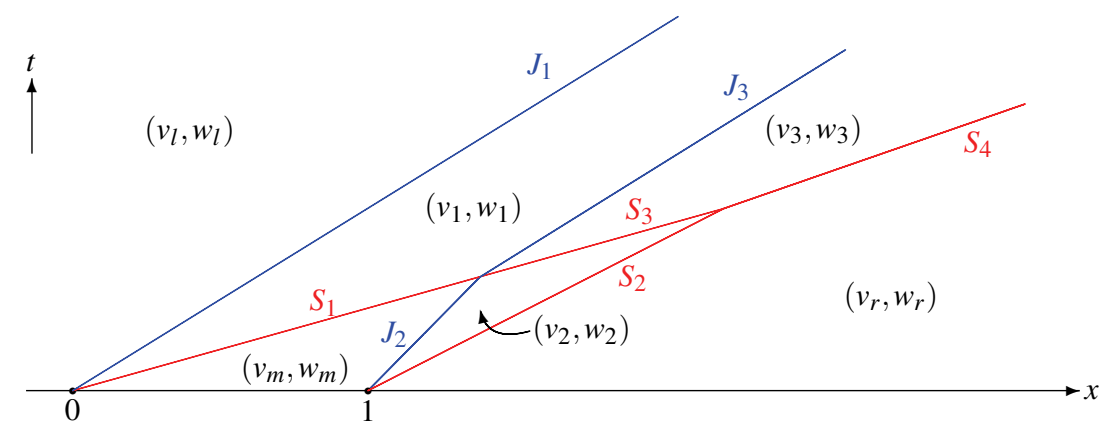

Fig.3.1. The global solution of the Cauchy problem (1.1) and (3.1) when $-1<v_{l}<v_{m}<v_{r}<0$.

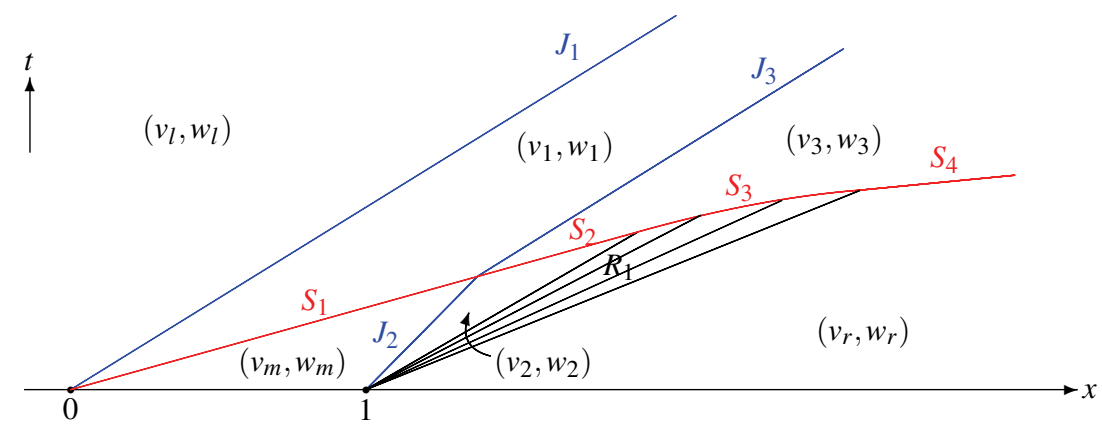

Fig.3.2. The global solution of the Cauchy problem (1.1) and (3.1) when $-1<v_{l}<v_{r}<v_{m}<0$.

The propagation speeds of $S_{1}$ and $J_{2}$ are $\sigma_{1}=\frac{1}{\left(1+v_{l}\right)\left(1+v_{m}\right)}$ and $\tau_{2}=\frac{1}{1+v_{m}}$ respectively, thus $S_{1}$ catches up with $J_{2}$ in finite time for $-1<v_{l}<v_{m}<0$. The intersection point of $S_{1}$ and $J_{2}$ is determined by

$$
\left\{\begin{array}{l}
x_{1}=\frac{t_{1}}{\left(1+v_{l}\right)\left(1+v_{m}\right)}, \\
x_{1}-1=\frac{t_{1}}{1+v_{m}},
\end{array}\right.
$$

which yields

$$
\left(x_{1}, t_{1}\right)=\left(-\frac{1}{v_{l}}, \frac{\left(1+v_{l}\right)\left(1+v_{m}\right)}{-v_{l}}\right) .
$$

At the intersection point $\left(x_{1}, t_{1}\right)$, a new Riemann problem is formed where the left state is $\left(v_{1}, w_{1}\right)$ and the right state is $\left(v_{2}, w_{2}\right)$. For $-1<v_{1}<v_{2}<0$ (namely $-1<v_{l}<v_{m}<0$ ), a new contact discontinuity $J_{3}$ and a new shock wave $S_{3}$ is generated and the immediate state between $J_{3}$ and $S_{3}$ can be derived from (3.4) that

$$
\left(v_{3}, w_{3}\right)=\left(v_{1}, \frac{v_{1} w_{2}}{v_{2}}\right)=\left(v_{l}, \frac{v_{l} w_{r}}{v_{r}}\right) .
$$

The propagation speeds of $J_{3}$ and $S_{3}$ are $\tau_{3}=\frac{1}{1+v_{l}}=\tau_{1}$ and $\sigma_{3}=\frac{1}{\left(1+v_{l}\right)\left(1+v_{m}\right)}=\sigma_{1}$. In other words, $J_{3}$ is parallel to $J_{1}$ and the shock wave keeps its direction unchanged when it passes 
through the contact discontinuity. Consequently, $S_{3}$ keeps up with $S_{2}$ in finite time and the intersection point of $S_{3}$ and $S_{2}$ is determined by

$$
\left\{\begin{array}{l}
x_{2}=\frac{t_{2}}{\left(1+v_{l}\right)\left(1+v_{m}\right)}, \\
x_{2}-1=\frac{t_{2}}{\left(1+v_{m}\right)\left(1+v_{r}\right)},
\end{array}\right.
$$

namely

$$
\left(x_{2}, t_{2}\right)=\left(\frac{1+v_{r}}{v_{r}-v_{l}}, \frac{\left(1+v_{l}\right)\left(1+v_{m}\right)\left(1+v_{r}\right)}{v_{r}-v_{l}}\right) .
$$

For $\frac{w_{3}}{v_{3}}=\frac{w_{r}}{v_{r}}, S_{3}$ and $S_{2}$ coalesce into a new shock wave $S_{4}$ whose propagation speed is $\sigma_{4}=$ $\frac{1}{\left(1+v_{l}\right)\left(1+v_{r}\right)}$.

If we take the limit $v_{l} \rightarrow(-1)^{+}$, then we have

$$
\lim _{v_{l} \rightarrow(-1)^{+}}\left(x_{1}, t_{1}\right)=\lim _{v_{l} \rightarrow(-1)^{+}}\left(x_{2}, t_{2}\right)=(1,0) .
$$

On the other hand, it is clear to see that all shock waves and contact discontinuities propagate with infinite speeds in the limit $v_{l} \rightarrow(-1)^{+}$situation. Thus, the Cauchy problem (1.1) and (3.1) has a zero shock wave emitting from the point $(0,0)$ in the limit $v_{l} \rightarrow(-1)^{+}$situation.

Case 3.2. $-1<v_{l}<v_{r}<v_{m}<0$

In this case, we consider that $J+R$ starts from $(1,0)$ when $-1<v_{l}<v_{r}<v_{m}<0$ is satisfied (see Fig.3.2). As in Case 3.1, the interaction of $S_{1}$ and $J_{2}$ at the point $\left(x_{1}, t_{1}\right)$ given by (3.6) generates a new contact discontinuity $J_{3}$ and a new shock wave $S_{2}$. The states $\left(v_{1}, w_{1}\right),\left(v_{2}, w_{2}\right)$ and $\left(v_{3}, w_{3}\right)$ can also be calculated by (3.4) and (3.7) respectively. Clearly, $S_{2}$ overtakes the wave back of $R_{1}$ in finite time and the intersection point $\left(x_{2}, t_{2}\right)$ is determined by

$$
\left\{\begin{array}{l}
x_{2}=\frac{t_{2}}{\left(1+v_{l}\right)\left(1+v_{m}\right)}, \\
x_{2}-1=\frac{t_{2}}{\left(1+v_{m}\right)^{2}},
\end{array}\right.
$$

namely

$$
\left(x_{2}, t_{2}\right)=\left(\frac{1+v_{m}}{v_{m}-v_{l}}, \frac{\left(1+v_{l}\right)\left(1+v_{m}\right)^{2}}{v_{m}-v_{l}}\right) .
$$

After the time $t_{2}, S_{2}$ begins to penetrate $R_{1}$ with a varying speed since it has a rarefaction wave on its right. During the process of penetration, it is denoted with $S_{3}$ which can be calculated by

$$
\left\{\begin{array}{l}
\sigma_{3}=\frac{d x}{d t}=\frac{1}{\left(1+v_{l}\right)(1+v)}, \\
x-1=\frac{t}{(1+v)^{2}}
\end{array}\right.
$$

with the initial condition (3.12), where $v$ varies from $v_{m}$ to $v_{r}$. Through solving it, we can obtain that

$$
\sqrt{x-1}=\frac{\sqrt{t}}{1+v_{l}}-\sqrt{\frac{v_{m}-v_{l}}{1+v_{l}}}
$$


It is clear that the shock wave accelerates during the process of penetration. The intersection point of $S_{3}$ and the wave front of $R_{1}$ is calculated by

$$
\left\{\begin{array}{l}
\sqrt{x_{3}-1}=\frac{\sqrt{t_{3}}}{1+v_{l}}-\sqrt{\frac{v_{m}-v_{l}}{1+v_{l}}}, \\
x_{3}-1=\frac{t_{3}}{\left(1+v_{r}\right)^{2}}
\end{array}\right.
$$

namely

$$
\left(x_{3}, t_{3}\right)=\left(1+\frac{\left(v_{m}-v_{l}\right)\left(1+v_{l}\right)}{\left(v_{r}-v_{l}\right)^{2}}, \frac{\left(v_{m}-v_{l}\right)\left(1+v_{l}\right)\left(1+v_{r}\right)^{2}}{\left(v_{r}-v_{l}\right)^{2}}\right) .
$$

After the time $t_{3}$, the shock wave is denoted with $S_{4}$ whose propagation speed is $\sigma_{4}=$ $\frac{1}{\left(1+v_{l}\right)\left(1+v_{r}\right)}$.

It follows from (3.6), (3.12) and (3.16) that

$$
\lim _{v_{l} \rightarrow(-1)^{+}}\left(x_{1}, t_{1}\right)=\lim _{v_{l} \rightarrow(-1)^{+}}\left(x_{2}, t_{2}\right)=\lim _{v_{l} \rightarrow(-1)+}\left(x_{3}, t_{3}\right)=(1,0) .
$$

In fact, all the waves become parts of the positive $x$-axis in the limit $v_{l} \rightarrow(-1)^{+}$situation. In this case, the Cauchy problem (1.1) and (3.1) has also a zero shock wave starting from the point $(0,0)$ in the limit $v_{l} \rightarrow(-1)^{+}$situation.

Case 3.3. $-1<v_{l}<v_{m}<0<v_{r}$

In this case, we consider that a delta shock wave $\delta S$ starts from $(1,0)$ when $-1<v_{l}<v_{m}<0<$ $v_{r}$ is satisfied (see Fig.3.3). The state $\left(v_{1}, w_{1}\right)$ can also be calculated by (3.4). Then, the intersection point of $S_{1}$ and $\delta S_{1}$ can be calculated by

$$
\left\{\begin{array}{l}
x_{1}=\frac{t_{1}}{\left(1+v_{l}\right)\left(1+v_{m}\right)} \\
x_{1}-1=\frac{t_{1}}{\left(1+v_{m}\right)\left(1+v_{r}\right)}
\end{array}\right.
$$

namely, we have

$$
\left(x_{1}, t_{1}\right)=\left(\frac{1+v_{r}}{v_{r}-v_{l}}, \frac{\left(1+v_{l}\right)\left(1+v_{m}\right)\left(1+v_{r}\right)}{v_{r}-v_{l}}\right) .
$$

In fact, the interaction of $S_{1}$ and $\delta S_{1}$ gives rise to a new delta shock wave $\delta S_{2}$ for $-1<v_{l}<$ $0<v_{r}$. Consequently, $\delta S_{2}$ meets with $J_{1}$ and a new interaction happens. The intersection point of $J_{1}$ and $\delta S_{2}$ can be calculated by

$$
\left\{\begin{array}{l}
x_{2}=\frac{t_{2}}{1+v_{l}}, \\
x_{2}-x_{1}=\frac{t_{2}-t_{1}}{\left(1+v_{l}\right)\left(1+v_{r}\right)},
\end{array}\right.
$$

with the initial condition (3.19). An easy calculation leads to

$$
\left(x_{2}, t_{2}\right)=\left(\frac{\left(v_{r}-v_{m}\right)\left(1+v_{r}\right)}{v_{r}\left(v_{r}-v_{l}\right)}, \frac{\left(v_{r}-v_{m}\right)\left(1+v_{l}\right)\left(1+v_{r}\right)}{v_{r}\left(v_{r}-v_{l}\right)}\right) .
$$




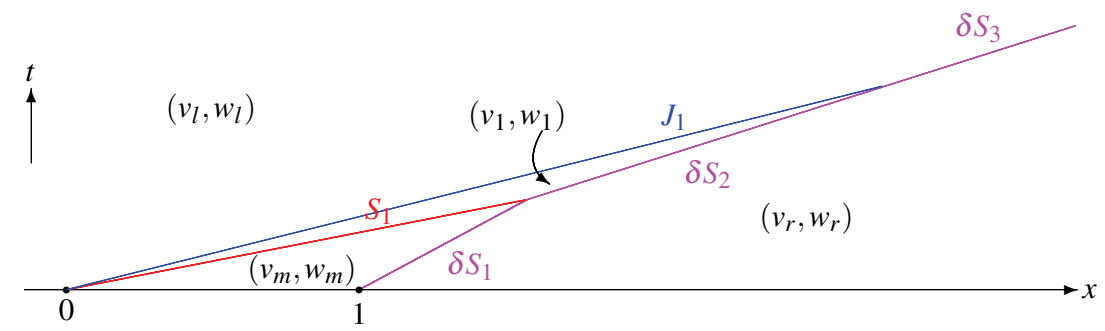

Fig.3.3. The global solution of the Cauchy problem (1.1) and (3.1) when $-1<v_{l}<v_{m}<0<v_{r}$.

After the time $t_{2}$, the delta shock wave continues to move forwards without changing its direction but has a different growth rate of the strength. Now, we are in a position to calculate the strength of the delta shock wave in the whole process in details. According to the different times, it follows from (2.10) that the strength of the delta shock wave can be calculated respectively by

$$
\begin{gathered}
\beta(t)=\frac{\left(v_{r} w_{m}-v_{m} w_{r}\right) t}{\left(1+v_{m}\right)\left(1+v_{r}\right)} \text { for } 0 \leq t \leq t_{1}, \\
\beta(t)=\beta\left(t_{1}\right)+\frac{v_{1}\left(v_{r} w_{m}-v_{m} w_{r}\right)\left(t-t_{1}\right)}{v_{m}\left(1+v_{l}\right)\left(1+v_{r}\right)} \text { for } t_{1} \leq t \leq t_{2}, \\
\beta(t)=\beta\left(t_{2}\right)+\frac{\left(v_{r} w_{l}-v_{l} w_{r}\right)\left(t-t_{2}\right)}{\left(1+v_{l}\right)\left(1+v_{r}\right)} \text { for } t \geq t_{2} .
\end{gathered}
$$

Analogously, it follows from (3.19) and (3.21) that

$$
\lim _{v_{l} \rightarrow(-1)^{+}}\left(x_{1}, t_{1}\right)=\lim _{v_{l} \rightarrow(-1)^{+}}\left(x_{2}, t_{2}\right)=(1,0) .
$$

Furthermore, for arbitrary $t>0$, we have $\lim _{v_{l} \rightarrow(-1)^{+}} \beta(t)=+\infty$ from (3.24). Thus, all the waves become parts of the positive $x$-axis and the zero delta shock wave is achieved in the limit $v_{l} \rightarrow$ $(-1)^{+}$situation. Hence, the Cauchy problem (1.1) and (3.1) has a zero shock wave in the interval $(0,1)$ in the positive $x$-axis and a zero delta shock wave starting from the point $(1,0)$ in the positive $x$-axis in the limit $v_{l} \rightarrow(-1)^{+}$situation.

Through the above analysis and discussion for the zero shock wave, we can summarize our results in the following theorem.

Theorem 3.1. When $-1<v_{l}<v_{m}<0$, the solution is a zero shock wave in the positive $x$-axis when $v_{r}<0$ and otherwise it is still a zero shock wave in the interval $(0,1)$ in the positive $x$-axis and then becomes a zero delta shock wave from the point $(1,0)$ in the positive $x$-axis when $v_{r}>0$ for the Cauchy problem (1.1) and (3.1) in the limit $v_{l} \rightarrow(-1)^{+}$situation .

\section{Analysis of Zero Delta Shock Wave}

In this section, we are interested in the situation that a zero delta shock wave interacts with the other elementary waves. In order to deal with it, we also consider the so-called double Riemann problem (1.1) and (3.1) and take $-1<v_{l}<-1+\varepsilon$ for sufficiently small $\varepsilon$ but require $v_{m}>0$. Thus, in the 
limit $v_{l} \rightarrow(-1)^{+}$situation, the Riemann solution to $(1.1)$ and $(1.2)$ at the point $(0,0)$ is a zero delta shock wave. In other words, we take the approximation of the zero delta shock wave in the Riemann solution at the point $(0,0)$. One can see that all the waves on the right become zero shock waves instantaneously in the limit $v_{l} \rightarrow(-1)^{+}$situation.

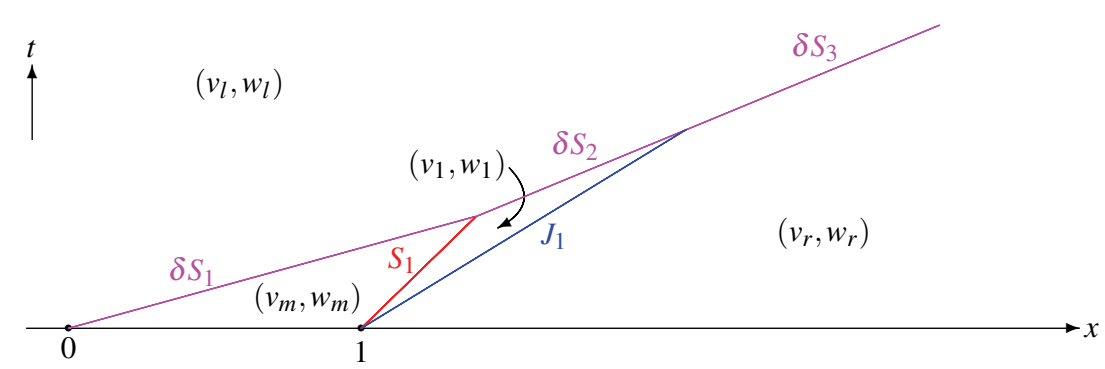

Fig.4.1. The global solution of the Cauchy problem (1.1) and (3.1) when $-1<v_{l}<0<v_{m}<v_{r}$.

Case 4.1. $-1<v_{l}<0<v_{m}<v_{r}$

In this case, we consider that $S+J$ starts from the point $(1,0)$ when $-1<v_{l}<0<v_{m}<v_{r}$ is satisfied (see Fig.4.1). The state $\left(v_{1}, w_{1}\right)$ is given by $\left(v_{1}, w_{1}\right)=\left(v_{r}, \frac{v_{r} w_{m}}{v_{m}}\right)$. The propagation speeds of $\delta S_{1}$ and $S$ are $\frac{1}{\left(1+v_{l}\right)\left(1+v_{m}\right)}$ and $\frac{1}{\left(1+v_{m}\right)\left(1+v_{r}\right)}$ respectively. Thus, the intersection point of $\delta S_{1}$ and $S_{1}$ can also be calculated by (3.19). Like as before, the interaction of $S_{1}$ and $\delta S_{1}$ also generates a new delta shock wave $\delta S_{2}$ with the propagation speed $\frac{1}{\left(1+v_{l}\right)\left(1+v_{r}\right)}$ for $-1<v_{l}<$ $0<v_{r}$. Consequently, $\delta S_{2}$ interacts with $J_{1}$ and the intersection point can be calculated by

$$
\left\{\begin{array}{l}
x_{2}-1=\frac{t_{2}}{1+v_{r}}, \\
x_{2}-x_{1}=\frac{t_{2}-t_{1}}{\left(1+v_{l}\right)\left(1+v_{r}\right)},
\end{array}\right.
$$

with the initial condition (3.19), which enables us to get

$$
\left(x_{2}, t_{2}\right)=\left(1+\frac{\left(v_{l}-v_{m}\right)\left(1+v_{l}\right)}{v_{l}\left(v_{r}-v_{l}\right)}, \frac{\left(v_{l}-v_{m}\right)\left(1+v_{l}\right)\left(1+v_{r}\right)}{v_{l}\left(v_{r}-v_{l}\right)}\right) .
$$

Similarly, the delta shock wave continues to move forwards without changing its direction when it passes through $J_{1}$ but has a different growth rate for the strength. According to the different times, the strength of the delta shock wave can be calculated respectively by

$$
\begin{gathered}
\beta(t)=\frac{\left(v_{m} w_{l}-v_{l} w_{m}\right) t}{\left(1+v_{l}\right)\left(1+v_{m}\right)} \quad \text { for } \quad 0 \leq t \leq t_{1}, \\
\beta(t)=\beta\left(t_{1}\right)+\frac{v_{r}\left(v_{m} w_{l}-v_{l} w_{m}\right)\left(t-t_{1}\right)}{v_{m}\left(1+v_{l}\right)\left(1+v_{r}\right)} \quad \text { for } \quad t_{1} \leq t \leq t_{2}, \\
\beta(t)=\beta\left(t_{2}\right)+\frac{\left(v_{r} w_{l}-v_{l} w_{r}\right)\left(t-t_{2}\right)}{\left(1+v_{l}\right)\left(1+v_{r}\right)} \quad \text { for } t \geq t_{2},
\end{gathered}
$$

in which $t_{1}$ and $t_{2}$ are given by (3.19) and (4.2) respectively.

Therefore, all the waves have infinite propagation speeds and the Cauchy problem (1.1) and (3.1) has a zero delta shock wave in the positive $x$-axis in the limit $v_{l} \rightarrow(-1)^{+}$situation. 


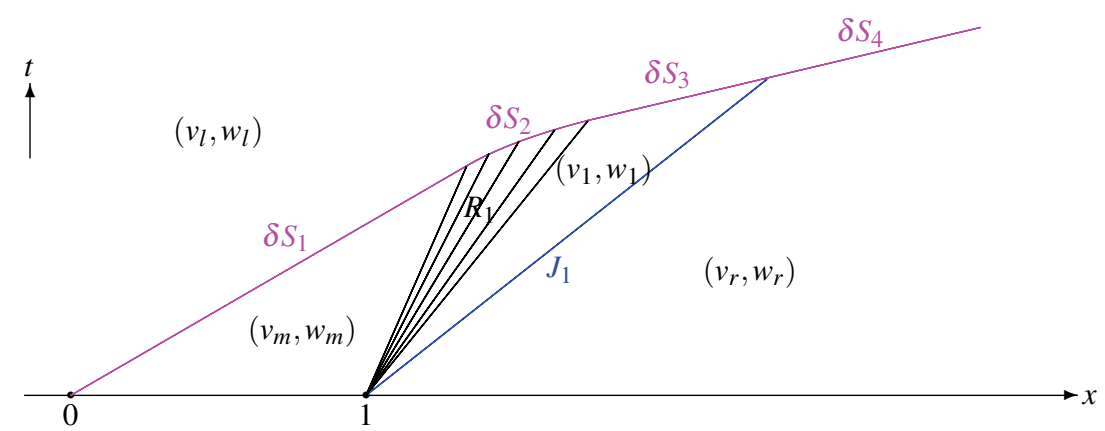

Fig.4.2. The global solution of the Cauchy problem (1.1) and (3.1) when $-1<v_{l}<0<v_{r}<v_{m}$.

Case 4.2. $-1<v_{l}<0<v_{r}<v_{m}$

In this case, we are concerned with the interaction of $\delta S$ and $R+J$ (see Fig.4.2). The occurrence of this case depends on the condition $-1<v_{l}<0<v_{r}<v_{m}$. The state between $R_{1}$ and $J_{1}$ is also $\left(v_{1}, w_{1}\right)=\left(v_{r}, \frac{v_{r} w_{m}}{v_{m}}\right)$. The propagation speed of $\delta S_{1}$ is $\sigma_{\delta}^{1}=\frac{1}{\left(1+v_{l}\right)\left(1+v_{m}\right)}$ and that of the wave back of $R_{1}$ is $\xi_{b}=\frac{1}{\left(1+v_{m}\right)^{2}}$. Thus $\delta S_{1}$ keeps up with the wave back of $R_{1}$ in finite time for $-1<v_{l}<0<v_{m}$. The intersection point $\left(x_{1}, t_{1}\right)$ of $\delta S_{1}$ and the wave back of $R_{1}$ can also be calculated by (3.11) and given by (3.12). The strength of the delta shock wave before the time $t_{1}$ can also be calculated by (4.3). Thus, the strength of $\delta S_{1}$ at the time $t_{1}$ is

$$
\beta\left(t_{1}\right)=\frac{\left(1+v_{m}\right)\left(v_{m} w_{l}-v_{l} w_{m}\right)}{v_{m}-v_{l}}
$$

After the time $t_{1}, \delta S_{1}$ begins to penetrate $R_{1}$. During the process of penetration, we denote it with $\delta S_{2}$ and use $\Gamma:\{x=x(t)\}$ to express, which has the same expression as (3.14).

In order to calculate the strength of the delta shock wave when it penetrates a rarefaction wave, we should adopt the method of splitting the delta function proposed by Nedeljkov and Oberguggenberger in [27], which has also been used in [34,35,38]. Now, we can construct the delta shock wave supported on the curve $\Gamma:\{x=x(t)\}$ of the form

$$
v(x, t)=\left\{\begin{array}{ll}
v_{l}, & x<x(t), \\
\sqrt{\frac{t}{x-1}}-1, & x>x(t),
\end{array} \quad w(x, t)=\left\{\begin{array}{ll}
w_{l}, & x<x(t) \\
\frac{w_{m}}{v_{m}}\left(\sqrt{\frac{t}{x-1}}-1\right), & x>x(t)
\end{array}\right\}+\beta_{-}(t) D_{\Gamma}^{-}+\beta_{+}(t) D_{\Gamma}^{+},\right.
$$

in which $\beta(t) D_{\Gamma}=\beta_{-}(t) D_{\Gamma}^{-}+\beta_{+}(t) D_{\Gamma}^{+}$is a split delta function supported on the curve $\Gamma$ and $\beta(t)=\beta_{-}(t)+\beta_{+}(t)$ denotes the strength of $\delta S_{2}$. Then, it follows from (4.7) that

$$
\begin{gathered}
w_{t}=\frac{w_{m} H}{2 v_{m} \sqrt{(x-1) t}}+\left(-\sigma_{\delta}^{2}\left(\frac{w_{m}}{v_{m}}\left(\sqrt{\frac{t}{x-1}}-1\right)-w_{l}\right)+\beta_{-}^{\prime}(t)+\beta_{+}^{\prime}(t)\right) \delta-\sigma_{\delta}^{2}\left(\beta_{-}(t)+\beta_{+}(t)\right) \delta^{\prime}, \\
\left(\frac{w}{1+v}\right)_{x}=\frac{-w_{m} H}{2 v_{m} \sqrt{(x-1) t}}+\left(\frac{w_{m}}{v_{m}}\left(1-\sqrt{\frac{x-1}{t}}\right)-\frac{w_{l}}{1+v_{l}}\right) \delta+\left(\frac{1}{1+v_{l}} \cdot \beta_{-}(t)+\sqrt{\frac{x-1}{t}} \cdot \beta_{+}(t)\right) \delta^{\prime},
\end{gathered}
$$


where all the $H, \delta, \delta^{\prime}$ are the functions of $x-x(t)$ in which $x(t)$ is given by (3.14). Substituting (4.8) and (4.9) into the second equation in (1.1) and then comparing the coefficients of $\delta$ and $\delta^{\prime}$, we have

$$
\begin{gathered}
-\sigma_{\delta}^{2}(t)\left(\frac{w_{m}}{v_{m}}\left(\sqrt{\frac{t}{x-1}}-1\right)-w_{l}\right)+\beta_{-}^{\prime}(t)+\beta_{+}^{\prime}(t)+\frac{w_{m}}{v_{m}}\left(1-\sqrt{\frac{x-1}{t}}\right)-\frac{w_{l}}{1+v_{l}}=0, \\
-\sigma_{\delta}^{2}(t)\left(\beta_{-}(t)+\beta_{+}(t)\right)+\frac{1}{1+v_{l}} \cdot \beta_{-}(t)+\sqrt{\frac{x-1}{t}} \cdot \beta_{+}(t)=0 .
\end{gathered}
$$

It can be derived from (3.13) that

$$
\sigma_{\delta}^{2}(t)=\frac{1}{\left(1+v_{l}\right)(1+v)}=\frac{1}{1+v_{l}} \cdot \sqrt{\frac{x-1}{t}},
$$

When $t \geq t_{1}$, by virtue of (3.14) and (4.12), it follows from (4.10) that

$$
\beta^{\prime}(t)=\beta_{-}^{\prime}(t)+\beta_{+}^{\prime}(t)=\frac{v_{m} w_{l}-v_{l} w_{m}}{\left(1+v_{l}\right) v_{m}} \cdot\left(1-\sqrt{\frac{x-1}{t}}\right)=\frac{v_{m} w_{l}-v_{l} w_{m}}{\left(1+v_{l}\right) v_{m}} \cdot\left(\frac{v_{l}}{1+v_{l}}+\sqrt{\frac{v_{m}-v_{l}}{\left(1+v_{l}\right) t}}\right) .
$$

Noticing the initial condition (4.6), it yields

$$
\beta(t)=\beta_{-}(t)+\beta_{+}(t)=\frac{v_{m} w_{l}-v_{l} w_{m}}{\left(1+v_{l}\right) v_{m}} \cdot\left(\frac{v_{l} t}{1+v_{l}}+2 \sqrt{\frac{\left(v_{m}-v_{l}\right) t}{1+v_{l}}}-1-v_{m}\right) .
$$

If the right state $\left(\sqrt{\frac{t}{x-1}}-1, \frac{w_{m}}{v_{m}}\left(\sqrt{\frac{t}{x-1}}-1\right)\right)$ of $\delta S_{2}$ arrives at $\left(v_{r}, \frac{v_{r} w_{m}}{v_{m}}\right)$, which is equivalent to $\sqrt{\frac{t}{x-1}}-1=v_{r}$, then $\delta S_{2}$ is just in time across the whole $R_{1}$ completely. Combining this with (3.14), we can obtain the intersection point of $\delta S_{1}$ and the wave back of $R_{1}$ as

$$
\left(x_{2}, t_{2}\right)=\left(1+\frac{\left(1+v_{l}\right)\left(v_{m}-v_{l}\right)}{\left(v_{r}-v_{l}\right)^{2}}, \frac{\left(1+v_{l}\right)\left(v_{m}-v_{l}\right)\left(1+v_{r}\right)^{2}}{\left(v_{r}-v_{l}\right)^{2}}\right) .
$$

The strength of $\delta S_{2}$ at $\left(x_{2}, t_{2}\right)$ can be calculated by (4.14).

Consequently, it is denoted with $\delta S_{3}$ and continues to move forwards and then interacts with $J_{1}$. The intersection point of $\delta S_{3}$ and $J_{1}$ can also be calculated by

$$
\left\{\begin{array}{l}
x_{3}-1=\frac{t_{3}}{1+v_{r}} \\
x_{3}-x_{2}=\frac{t_{3}-t_{2}}{\left(1+v_{l}\right)\left(1+v_{r}\right)}
\end{array}\right.
$$

with the initial condition (4.15), which enables us to have

$$
\left(x_{3}, t_{3}\right)=\left(1+\frac{\left(1+v_{l}\right)\left(v_{m}-v_{l}\right)}{v_{l}\left(v_{l}-v_{r}\right)}, \frac{\left(1+v_{l}\right)\left(1+v_{r}\right)\left(v_{m}-v_{l}\right)}{v_{l}\left(v_{l}-v_{r}\right)}\right) .
$$

Similarly, the delta shock wave continues to move forwards without changing its direction when it passes through $J_{1}$ but has a different growth rate for the strength. More precisely, we have

$$
\beta(t)=\beta\left(t_{2}\right)+\frac{v_{r}\left(v_{m} w_{l}-v_{l} w_{m}\right)\left(t-t_{2}\right)}{v_{m}\left(1+v_{l}\right)\left(1+v_{r}\right)} \quad \text { for } \quad t_{2} \leq t \leq t_{3},
$$




$$
\beta(t)=\beta\left(t_{3}\right)+\frac{\left(v_{r} w_{l}-v_{l} w_{r}\right)\left(t-t_{3}\right)}{\left(1+v_{l}\right)\left(1+v_{r}\right)} \quad \text { for } \quad t \geq t_{3}
$$

in which $t_{2}$ and $t_{3}$ are given by (4.15) and (4.17) respectively.

If we take the limit $v_{l} \rightarrow(-1)^{+}$in (3.12), (4.15) and (4.17), then we can see that all the points $\left(x_{1}, t_{1}\right),\left(x_{2}, t_{2}\right)$ and $\left(x_{3}, t_{3}\right)$ tend to $(1,0)$. In the limit $v_{l} \rightarrow(-1)^{+}$situation, the delta shock wave passes through $R_{1}$ and $J_{1}$ with the infinite propagation speed and the Cauchy problem (1.1) and (3.1) has also a zero delta shock wave in the positive $x$-axis.

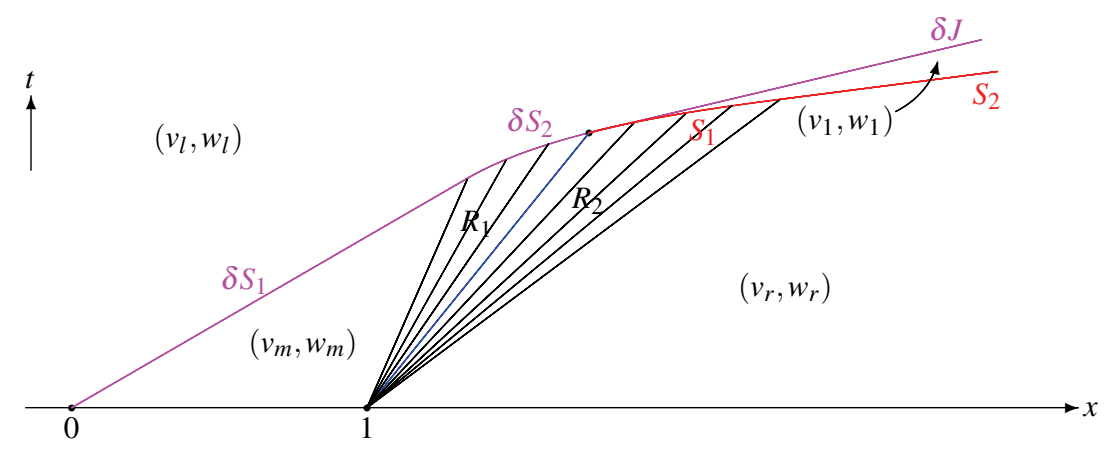

Fig.4.3. The global solution of the Cauchy problem (1.1) and (3.1) when $-1<v_{l}<v_{r}<0<v_{m}$.

Case 4.3. $-1<v_{l}<v_{r}<0<v_{m}$

In this case, we are concerned with the interaction of $\delta S$ and $R_{1}+R_{2}$ when the condition $-1<v_{l}<v_{r}<0<v_{m}$ holds (see Fig.4.3). In the beginning, the situation in this case is identical with that of Case 4.2. In other words, $\delta S_{1}$ catches up with the wave back of $R_{1}$ in finite time and the intersection point of them is given by (3.12) and the strength of the delta shock wave at the intersection point is also given by (4.6). Consequently, it begins to penetrate $R_{1}$ and be denoted with $\delta S_{2}$ during the process of penetration. In this process, the curve $\Gamma$ of $\delta S_{2}$ can also be expressed by (3.14) and the strength of $\delta S_{2}$ can also be calculated by (4.14) until it arrives at the line $x-1=t$.

It is clear to see that the intersection point of the curve of $\delta S_{2}$ and the line $x-1=t$ can be calculated by

$$
\left\{\begin{array}{l}
\sqrt{x_{2}-1}=\frac{\sqrt{t_{2}}}{1+v_{l}}-\sqrt{\frac{v_{m}-v_{l}}{1+v_{l}}} . \\
x_{2}-1=t_{2}
\end{array}\right.
$$

which implies that

$$
\left(x_{2}, t_{2}\right)=\left(\frac{v_{m}-v_{l}+v_{l} v_{m}}{v_{l}^{2}}, \frac{\left(1+v_{l}\right)\left(v_{m}-v_{l}\right)}{v_{l}^{2}}\right) .
$$

Then, it follows from (4.14) that the strength of delta shock wave at the time $t_{2}$ is given by

$$
\beta\left(t_{2}\right)=\frac{v_{m} w_{l}-v_{l} w_{m}}{-v_{l}} .
$$

In what follows, we can see that the delta shock wave supported by $\Gamma$ is an overcompressive wave only up to the point $\left(x_{2}, t_{2}\right)$. It is remarkable that the state $(v, w)$ in $R_{2}$ is calculated by $\frac{w}{v}=\frac{w_{r}}{v_{r}}$, which is obviously different from $\frac{w}{v}=\frac{w_{m}}{v_{m}}$ in $R_{1}$ on the right-hand side of the line $x-1=t$. 
Especially, we can see that $v<0$ in $R_{2}$. A new local Riemann problem containing a Dirac delta measure at the intersection point $\left(x_{2}, t_{2}\right)$ is formulated as

$$
\left.v\right|_{t=t_{2}}=\left\{\left.\begin{array}{ll}
v_{l}, & x<x_{2}, \\
\eta, & x>x_{2},
\end{array} \quad w\right|_{t=t_{2}}=\left\{\begin{array}{ll}
w_{l}, & x<x_{2} \\
\frac{\eta w_{r}}{v_{r}}, & x>x_{2}
\end{array}\right\}+\beta\left(t_{2}\right) \delta_{\left(x_{2}, t_{2}\right)},\right.
$$

where we suppose that $\eta(<0)$ is an approximation of zero and satisfies $\frac{x-1}{t}=\frac{1}{(1+\eta)^{2}}$, namely we assume that $R_{2}$ is approximated by a set of non-physical shock waves.

Actually, the solution of the local Riemann problem (1.1) and (4.23) can be constructed in the form:

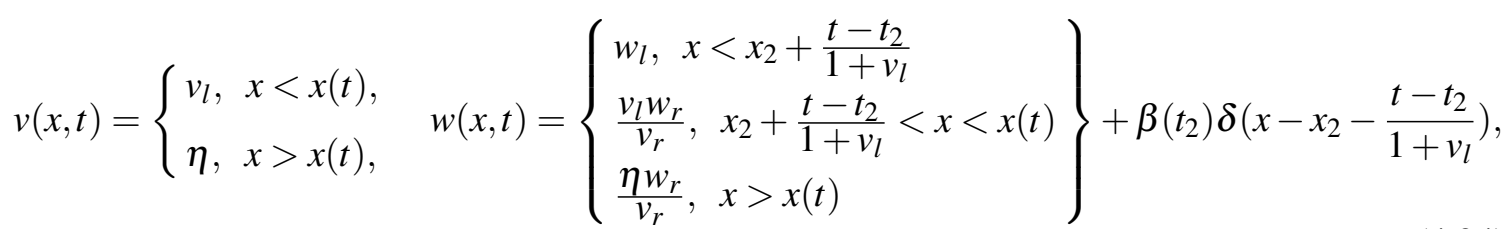

where the shock wave curve $x=x(t)$ can be expressed as $x(t)=x_{2}+\frac{t-t_{2}}{\left(1+v_{l}\right)(1+\eta)}$ in the local neighborhood of $\left(x_{2}, t_{2}\right)$ for the sufficient approximation $\eta(<0)$ of zero.

Now, one is in a position to check that (4.24) is indeed the weak solution of the local Riemann problem (1.1) and (4.23). For every $\varphi \in C_{0}^{\infty}\left(R \times R_{+}\right)$, it is clear to see that

$$
\left\{\begin{array}{l}
\left\langle v_{t}+\left(\frac{v}{1+v}\right)_{x}, \varphi\right\rangle=0 \\
\left\langle w_{t}+\left(\frac{w}{1+v}\right)_{x}, \varphi\right\rangle=0
\end{array}\right.
$$

hold if $\operatorname{supp} \varphi \cap\left\{(x, t) \mid x=x_{2}+\frac{t-t_{2}}{1+v_{l}}, t>t_{2}\right\}=\emptyset$. Otherwise, we can see that (4.24) is also the weak solution of (1.1) and (4.23) near the support of the Dirac delta measure. For the first equation in (1.1), the result obviously holds since it does not contain $w$ and no singularity appears in it. For the second equation in (1.1), through substituting (4.24) into it, we can see that

$$
w_{t}+\left(\frac{w}{1+v}\right)_{x}=-\frac{1}{1+v_{l}}\left(\left(\frac{v_{l} w_{r}}{v_{r}}-w_{l}\right) \delta+\beta\left(t_{2}\right) \delta^{\prime}\right)+\left(\frac{v_{l} w_{r}}{v_{r}\left(1+v_{l}\right)}-\frac{w_{l}}{1+v_{l}}\right) \delta+\frac{\beta\left(t_{2}\right)}{1+v_{l}} \delta^{\prime}=0
$$

holds near the line $x=x_{2}+\frac{t-t_{2}}{1+v_{l}}$ for $t \geq t_{2}$ in the weak (or distributional) sense.

Thus, we can see that the Dirac delta function is now supported on the contact discontinuity line $x=x_{2}+\frac{t-t_{2}}{1+v_{l}}$ which is called as the delta contact discontinuity $\delta J$ in [27,35]. When $t>t_{2}, \delta S_{2}$ is decomposed into $\delta J$ and $S_{1}$ with the middle state $\left(v_{1}, w_{1}\right)=\left(v_{l}, \frac{v_{l} w_{r}}{v_{r}}\right)$ between them. Consequently, $\delta J$ continues to move forwards with the constant speed $\frac{1}{1+v_{l}}$ and the invariant strength $\beta\left(t_{2}\right)$. On the other hand, $S_{1}$ continues to penetrate $R_{2}$ also with a varying propagation speed. It is noticed that $S_{1}$ has the same propagation speed with $\delta J$ at $\left(x_{2}, t_{2}\right)$ for the state on the right-hand side of the point satisfies $v=0$. In other words, $S_{1}$ and $\delta J$ are tangential at the point $\left(x_{2}, t_{2}\right)$.

After the time $t_{2}$, the state on the left-hand side of $S_{1}$ is $\left(v_{1}, w_{1}\right)=\left(v_{l}, \frac{v_{l} w_{r}}{v_{r}}\right)$ and the state on the right-hand side of $S_{1}$ is $(v, w)=\left(\sqrt{\frac{t}{x-1}}-1, \frac{w_{r}}{v_{r}}\left(\sqrt{\frac{t}{x-1}}-1\right)\right)$ which corresponds to the state on the characteristic line in $R_{2}$. Thus, the curve of $S_{1}$ is also determined by (3.13) with the initial 
condition (3.12). Here we notice that the initial point $\left(x_{2}, t_{2}\right)$ also lies on the curve $\Gamma$ given by (3.14). Thus, the shock wave curve $S_{1}$ is a continuation of the curve $\Gamma$, for the propagation speed of $S_{1}$ is determined by the value $v$ only.

For $-1<v_{l}<v_{r}<0$, it is clear to see that $S_{1}$ is able to penetrate $R_{2}$ completely. The intersection point of $S_{1}$ and the wave front of $R_{2}$ can be calculated by

$$
\left\{\begin{array}{l}
\sqrt{x_{3}-1}=\frac{\sqrt{t_{3}}}{1+v_{l}}-\sqrt{\frac{v_{m}-v_{l}}{1+v_{l}}} \\
x_{3}-1=\frac{t_{3}}{\left(1+v_{r}\right)^{2}}
\end{array}\right.
$$

which means that

$$
\left(x_{3}, t_{3}\right)=\left(1+\frac{\left(1+v_{l}\right)\left(v_{m}-v_{l}\right)}{\left(v_{r}-v_{l}\right)^{2}}, \frac{\left(1+v_{l}\right)\left(1+v_{r}\right)^{2}\left(v_{m}-v_{l}\right)}{\left(v_{r}-v_{l}\right)^{2}}\right) .
$$

Similarly, if we take the limit $v_{l} \rightarrow(-1)^{+}$in (3.12), (4.21) and (4.28), then we can also see that all the points $\left(x_{1}, t_{1}\right),\left(x_{2}, t_{2}\right)$ and $\left(x_{3}, t_{3}\right)$ tend to $(1,0)$. In the limit $v_{l} \rightarrow(-1)^{+}$situation, the delta shock wave passes through $R_{1}$ and $R_{2}$ with the infinite propagation speed and the Cauchy problem (1.1) and (3.1) has also a zero delta shock wave in the positive $x$-axis. But for $x \geq 1$ on the positive $x$-axis, the zero delta shock wave is the superposition of a zero delta contact discontinuity and a zero shock wave.

Hence, we can also summarize our results for the zero delta shock wave in the limit $v_{l} \rightarrow(-1)^{+}$ situation in the following theorem.

Theorem 4.1. If the initial condition (3.1) satisfies $-1<v_{l}<0<v_{m}$ and $v_{l}<v_{r}$, then a zero delta shock wave generates at the point $(0,0)$ and keeps in the positive $x$-axis in the limit $v_{l} \rightarrow(-1)^{+}$ situation for the Cauchy problem (1.1) and (3.1).

\section{Analysis of Zero Rarefaction Wave}

In this section, we turn our attentions to the situation that the zero rarefaction wave interacts with the other elementary waves. In order to investigate it, we take $-1<v_{m}<-1+\varepsilon$ for sufficiently small $\varepsilon$, and then we pass to the limit $v_{m} \rightarrow(-1)^{+}$. Thus, in the limit $v_{m} \rightarrow(-1)^{+}$situation, the Riemann solution to (1.1) and (1.2) at the point $(0,0)$ has a zero rarefaction wave. This is to say, we take the approximation of the zero rarefaction wave in the Riemann solution at the point $(0,0)$.

Our discussion should be divided into two parts according to $v_{l}>0$ or not. To be more precise, if we take $-1<v_{m}<-1+\varepsilon$ for sufficiently small $\varepsilon$, then the Riemann solution at the point $(0,0)$ is $R_{1}+R_{2}$ when $v_{l}>0$, otherwise the Riemann solution at the point $(0,0)$ is $J+R$ when $v_{l}<0$. Now we first consider the situation $v_{l}>0$ and then consider the situation $v_{l}<0$.

Case 5.1. $-1<v_{m}<v_{r}<0<v_{l}$

In this case, we are concerned with the interaction of $R_{1}+R_{2}$ and $J+S$ under the condition $-1<v_{m}<v_{r}<0<v_{l}$ (see Fig.5.1). The propagation speed of the wave front of $R_{1}$ and $J_{1}$ are $\xi_{f}=\frac{1}{\left(1+v_{m}\right)^{2}}$ and $\tau_{1}=\frac{1}{1+v_{m}}$ respectively. For $-1<v_{m}<0$, it is clear that the wave front of $R_{1}$ overtakes $J_{1}$ in finite time and the intersection point is determined by 


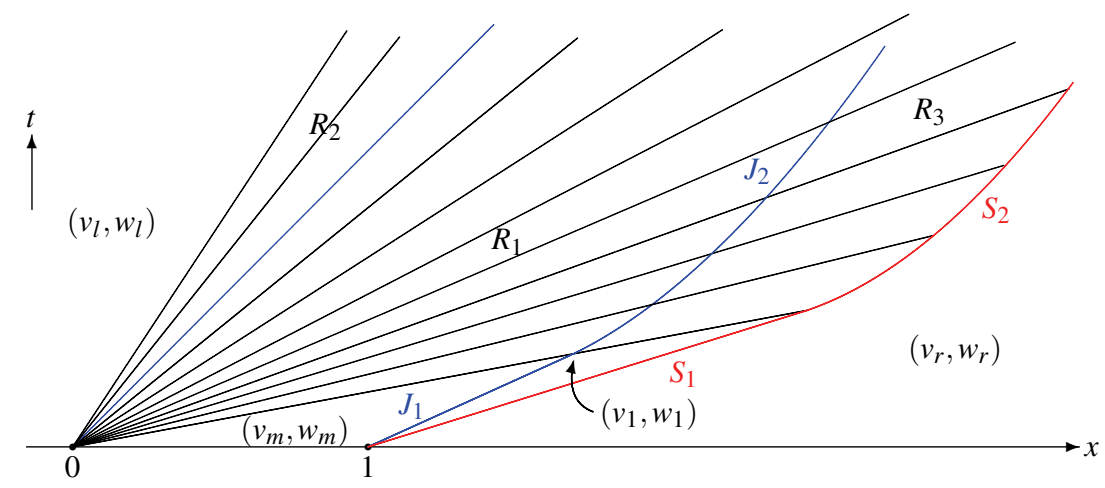

Fig.5.1. The global solution of the Cauchy problem (1.1) and (3.1) when $-1<v_{m}<v_{r}<0<v_{l}$.

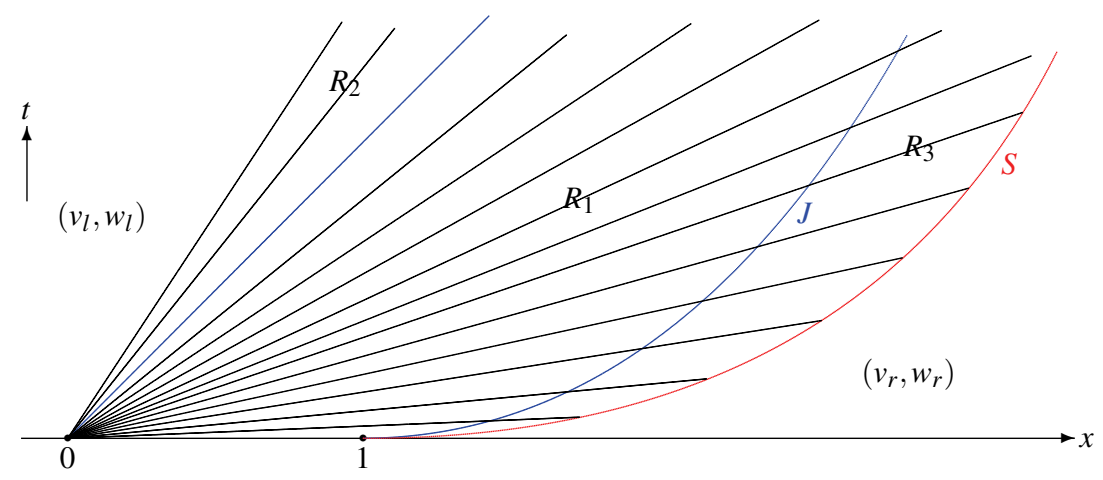

Fig.5.2. The limit $v_{m} \rightarrow(-1)^{+}$of solution of the Cauchy problem (1.1) and (3.1) when $-1<v_{m}<$ $v_{r}<0<v_{l}$.

$$
\left\{\begin{array}{l}
x_{1}=\frac{t_{1}}{\left(1+v_{m}\right)^{2}}, \\
x_{1}-1=\frac{t_{1}}{1+v_{m}},
\end{array}\right.
$$

which yields

$$
\left(x_{1}, t_{1}\right)=\left(\frac{1}{-v_{m}}, \frac{\left(1+v_{m}\right)^{2}}{-v_{m}}\right) .
$$

Consequently, $J_{1}$ begins to penetrate $R_{1}$ with a varying propagation speed. During the process of penetration, we denote it with $J_{2}$ which is determined by

$$
\frac{d x}{d t}=\frac{1}{1+v}=\sqrt{\frac{x}{t}}
$$

with the initial condition (5.2). An easy computation leads to

$$
\sqrt{x}=\sqrt{t}+\sqrt{-v_{m}} .
$$

It is clear that $J_{2}$ decelerates when passing through $R_{1}$. But we notice that the line $x=t$ cannot keep up with $J_{2}$ forever. Thus, $J_{2}$ has the line $x=t$ as its asymptotic and is not able to enter the region of $R_{1}$. 
On the other hand, $R_{1}$ passes through $J_{2}$ and then is denoted with $R_{3}$ after penetration. Here we should emphasize a fact that the propagation direction of the rarefaction wave is unchanged during the process of penetration since the value $v=\sqrt{\frac{t}{x}}-1$ is unchanged when across $J_{2}$. However, the value $w$ varies from $w=\frac{v w_{m}}{v_{m}}$ in $R_{1}$ to $w=\frac{v w_{r}}{v_{r}}$ in $R_{3}$ when across $J_{2}$.

Now let us consider the interaction of $R_{3}$ and $S_{1}$. The propagation speed of the wave front of $R_{3}$ is also $\xi=\frac{1}{\left(1+v_{m}\right)^{2}}$ and that of $S_{1}$ is $\sigma_{1}=\frac{1}{\left(1+v_{m}\right)\left(1+v_{r}\right)}$. For $-1<v_{m}<v_{r}<0$, it is easy to see that the wave front of $R_{3}$ is also able to catch up with $S_{1}$ in finite time and the intersection point can be calculated by

$$
\left\{\begin{array}{l}
x_{2}=\frac{t_{2}}{\left(1+v_{m}\right)^{2}}, \\
x_{2}-1=\frac{t_{2}}{\left(1+v_{m}\right)\left(1+v_{r}\right)},
\end{array}\right.
$$

which implies that

$$
\left(x_{2}, t_{2}\right)=\left(\frac{1+v_{r}}{v_{r}-v_{m}}, \frac{\left(1+v_{m}\right)^{2}\left(1+v_{r}\right)}{v_{r}-v_{m}}\right) .
$$

After the time $t_{2}, S_{1}$ begins to penetrate $R_{3}$ and is denoted with $S_{2}$ during the process of penetration, whose curve can be calculated by

$$
\frac{d x}{d t}=\frac{1}{(1+v)\left(1+v_{r}\right)}=\frac{1}{1+v_{r}} \cdot \sqrt{\frac{x}{t}}
$$

with the initial condition (5.6). An easy computation leads to

$$
\sqrt{x}=\frac{\sqrt{t}}{1+v_{r}}+\sqrt{\frac{v_{r}-v_{m}}{1+v_{r}}} .
$$

It is clear that $S_{2}$ also decelerates when passing through $R_{3}$. For $v_{r}<0, S_{2}$ cannot penetrate $R_{3}$ completely and finally has the characteristic line $x=\frac{t}{\left(1+v_{r}\right)^{2}}$ in $R_{3}$ as its asymptote.

Analogously, if we take the limit $v_{m} \rightarrow(-1)^{+}$in (5.2) and (5.6), then we can see that both the points $\left(x_{1}, t_{1}\right)$ and $\left(x_{2}, t_{2}\right)$ tend to $(1,0)$. For the Cauchy problem (1.1) and (3.1), the zero rarefaction wave only exists in the interval $[0,1]$ in the positive $x$-axis in the limit $v_{m} \rightarrow(-1)^{+}$situation since the contact discontinuity and the shock wave begin to penetrate the rarefaction wave from the point $(1,0)$ (see Fig.5.2).

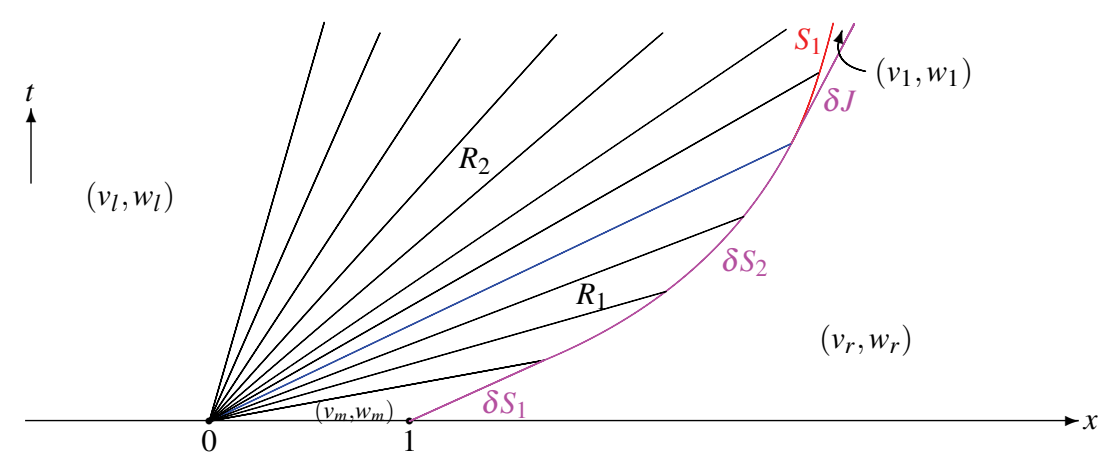

Fig.5.3. The global solution of the Cauchy problem (1.1) and (3.1) when $-1<v_{m}<0<v_{r}<v_{l}$. 
Case 5.2. $-1<v_{m}<0<v_{r}<v_{l}$

In this case, we are concerned with the interaction of $R_{1}+R_{2}$ and $\delta S$ under the condition $-1<$ $v_{m}<0<v_{r}<v_{l}$ (see Fig.5.3). It is clear that the wave front of $R_{1}$ overtakes $\delta S_{1}$ in finite time and the intersection point $\left(x_{1}, t_{1}\right)$ is calculated by (5.5) and given by (5.6). In other words, we only need use $\left(x_{1}, t_{1}\right)$ to replace of $\left(x_{2}, t_{2}\right)$ in (5.5) and (5.6). It is clear that the strength of $\delta S_{1}$ at the point $\left(x_{1}, t_{1}\right)$ can be calculated by

$$
\beta\left(t_{1}\right)=\frac{\left(v_{r} w_{m}-v_{m} w_{r}\right) t_{1}}{\left(1+v_{m}\right)\left(1+v_{r}\right)}=\frac{\left(1+v_{m}\right)\left(v_{r} w_{m}-v_{m} w_{l}\right)}{v_{r}-v_{m}} .
$$

Consequently, $\delta S_{1}$ begins to penetrate $R_{1}$ and is denoted with $\delta S_{2}$ during the process of penetration. The curve of $\delta S_{2}$ can also be calculated by (5.7) and expressed by (5.8) until it meets the line $x=t$ at the point

$$
\left(x_{2}, t_{2}\right)=\left(\frac{\left(v_{r}-v_{m}\right)\left(1+v_{r}\right)}{v_{r}}, \frac{\left(v_{r}-v_{m}\right)\left(1+v_{r}\right)}{v_{r}}\right) .
$$

As in Case 4.2, in order to calculate the strength of the delta shock wave during the process of penetration, we also use the method of splitting the delta function and construct the delta shock wave supported on the curve (5.8) of $\delta S_{2}$ in the form

$$
v(x, t)=\left\{\begin{array}{ll}
\sqrt{\frac{t}{x}}-1, & x<x(t), \\
v_{r}, & x>x(t),
\end{array} \quad w(x, t)=\left\{\begin{array}{ll}
\frac{w_{m}}{v_{m}}\left(\sqrt{\frac{t}{x}}-1\right), & x<x(t) \\
w_{r}, & x>x(t)
\end{array}\right\}+\beta_{-}(t) D_{\Gamma}^{-}+\beta_{+}(t) D_{\Gamma}^{+} .\right.
$$

Then, it follows from (5.11) that

$$
\begin{gathered}
w_{t}=-\frac{w_{m} H}{2 v_{m} \sqrt{x t}}+\left(-\sigma_{\delta}^{2}\left(w_{r}-\frac{w_{m}}{v_{m}}\left(\sqrt{\frac{t}{x}}-1\right)\right)+\beta_{-}^{\prime}(t)+\beta_{+}^{\prime}(t)\right) \delta-\sigma_{\delta}^{2}\left(\beta_{-}(t)+\beta_{+}(t)\right) \delta^{\prime}, \\
\left(\frac{w}{1+v}\right)_{x}=\frac{w_{m} H}{2 v_{m} \sqrt{x t}}+\left(\frac{w_{r}}{1+v_{r}}-\frac{w_{m}}{v_{m}}\left(1-\sqrt{\frac{x}{t}}\right)\right) \delta+\left(\sqrt{\frac{x}{t}} \cdot \beta_{-}(t)+\frac{1}{1+v_{r}} \cdot \beta_{+}(t)\right) \delta^{\prime}
\end{gathered}
$$

where all the $H, \delta, \delta^{\prime}$ are also the functions of $x-x(t)$ in which $x(t)$ is given by (5.8). Substituting (5.12) and (5.13) into the second equation in (1.1) and comparing the coefficients of $\delta$ and $\delta^{\prime}$, we have

$$
\begin{gathered}
\left(-\sigma_{\delta}^{2}\left(w_{r}-\frac{w_{m}}{v_{m}}\left(\sqrt{\frac{t}{x}}-1\right)\right)+\beta_{-}^{\prime}(t)+\beta_{+}^{\prime}(t)+\frac{w_{r}}{1+v_{r}}-\frac{w_{m}}{v_{m}}\left(1-\sqrt{\frac{x}{t}}\right)=0,\right. \\
-\sigma_{\delta}^{2}(t)\left(\beta_{-}(t)+\beta_{+}(t)\right) \sqrt{\frac{x}{t}} \cdot \beta_{-}(t)+\frac{1}{1+v_{r}} \cdot \beta_{+}(t)=0 .
\end{gathered}
$$

When $t \geq t_{1}$, by virtue of (5.7) and (5.8), it follows from (5.14) that

$$
\beta^{\prime}(t)=\beta_{-}^{\prime}(t)+\beta_{+}^{\prime}(t)=\frac{v_{r} w_{m}-v_{m} w_{r}}{v_{m}\left(1+v_{r}\right)} \cdot\left(1-\sqrt{\frac{x}{t}}\right)=\frac{v_{r} w_{m}-v_{m} w_{r}}{v_{m}\left(1+v_{r}\right)} \cdot\left(\frac{v_{r}}{1+v_{r}}-\sqrt{\frac{v_{r}-v_{m}}{\left(1+v_{r}\right) t}}\right) .
$$


Then, we have

$$
\beta(t)=\beta_{-}(t)+\beta_{+}(t)=\frac{v_{r} w_{m}-v_{m} w_{r}}{v_{m}\left(1+v_{r}\right)} \cdot\left(\frac{v_{r} t}{1+v_{r}}-2 \sqrt{\frac{\left(v_{r}-v_{m}\right) t}{1+v_{r}}}+1+v_{m}\right),
$$

in which the initial condition (5.9) has been used.

After the time $t_{2}$, the situation is similar to that in Case 4.3, namely $\delta S_{2}$ is also decomposed into a shock wave $S_{1}$ and a delta contact discontinuity $\delta J$ with the middle state $\left(v_{1}, w_{1}\right)=\left(v_{r}, \frac{v_{r} w_{l}}{v_{l}}\right)$ between them. Consequently, $\delta J$ continues to move forwards with the constant speed $\frac{1}{1+v_{r}}$ and the invariant strength $\beta\left(t_{2}\right)$. On the other hand, $S_{1}$ is tangential with $\delta J$ at the point $\left(x_{2}, t_{2}\right)$ and then continues to penetrate $R_{2}$ also with a varying propagation speed. With the same procedure as before, we can see that the shock wave curve of $S_{1}$ is a continuation of the curve (5.8) of $\delta S_{2}$. For $0<v_{r}<v_{l}$, we can see that $S_{1}$ cannot penetrate $R_{2}$ completely and finally has the characteristic line $x=\frac{t}{\left(1+v_{r}\right)^{2}}$ in $R_{2}$ as its asymptote.

For the Cauchy problem (1.1) and (3.1), the zero rarefaction wave also only exists in the interval $[0,1]$ in the positive $x$-axis in the limit $v_{m} \rightarrow(-1)^{+}$situation since the delta shock wave begins to penetrate the rarefaction wave from the point $(1,0)$.

Case 5.3. $-1<v_{m}<0<v_{l}<v_{r}$

In this case, it is also the interaction problem for $R_{1}+R_{2}$ and $\delta S$ when the condition $-1<$ $v_{m}<0<v_{r}<v_{l}$ is satisfied. The situation in this case is similar to that in Case 5.2 and the difference only lies in that $S_{1}$ is able to penetrate $R_{2}$ completely and finally has the propagation speed $\frac{1}{\left(1+v_{l}\right)\left(1+v_{r}\right)}$ after penetration. The details are omitted and left to the readers.

Case 5.4. $-1<v_{m}<v_{l}<0<v_{r}$

In this case, we are concerned with the interaction of $J+R$ and $\delta S$ when the condition $-1<$ $v_{m}<v_{l}<0<v_{r}$ is satisfied. The situation of wave interaction is analogous to that in Case 4.2 but the limit situation is different from that in Case 4.2. If we take the limit $v_{m} \rightarrow(-1)^{+}$, then $\delta S$ is tangential to the positive $x$-axis at the point $(1,0)$ and consequently penetrates $R$ completely. Finally, it continues to move forwards without changing its direction when it passes through $J$.

Case 5.5. $-1<v_{m}<v_{l}<v_{r}<0$

In this case, we are concerned with the interaction of $J+R$ and $J+S$ when the condition $-1<$ $v_{m}<v_{l}<v_{r}<0$ is satisfied. In the beginning, the situation is similar to that in Case 5.1. More precisely, the contact discontinuity $J_{2}$ passes through the rarefaction wave $R_{1}$ with a varying speed and the direction of rarefaction wave is also unchanged. During the process of penetration, the curve of contact discontinuity $J_{3}$ can also be calculated by (5.4). But here the contact discontinuity $J_{3}$ is able to penetrate the rarefaction wave $R_{1}$ completely and finally has the propagation speed $\frac{1}{1+v_{l}}$, which is parallel to $J_{1}$. On the other hand, the shock wave $S_{1}$ also penetrates the rarefaction wave $R_{2}$ and the curve of it can also be calculated by (5.8). Here the shock wave $S_{1}$ is able to penetrate the rarefaction wave $R_{2}$ completely and finally has the propagation speed $\frac{1}{\left(1+v_{l}\right)\left(1+v_{r}\right)}$.

We can depict Figure 5.4 to explain this case, in which

$$
\left(v_{1}, w_{1}\right)=\left(v_{l}, \frac{v_{l} w_{m}}{v_{m}}\right), \quad\left(v_{2}, w_{2}\right)=\left(v_{m}, \frac{v_{m} w_{r}}{v_{r}}\right) \quad \text { and } \quad\left(v_{3}, w_{3}\right)=\left(v_{l}, \frac{v_{l} w_{r}}{v_{r}}\right) .
$$




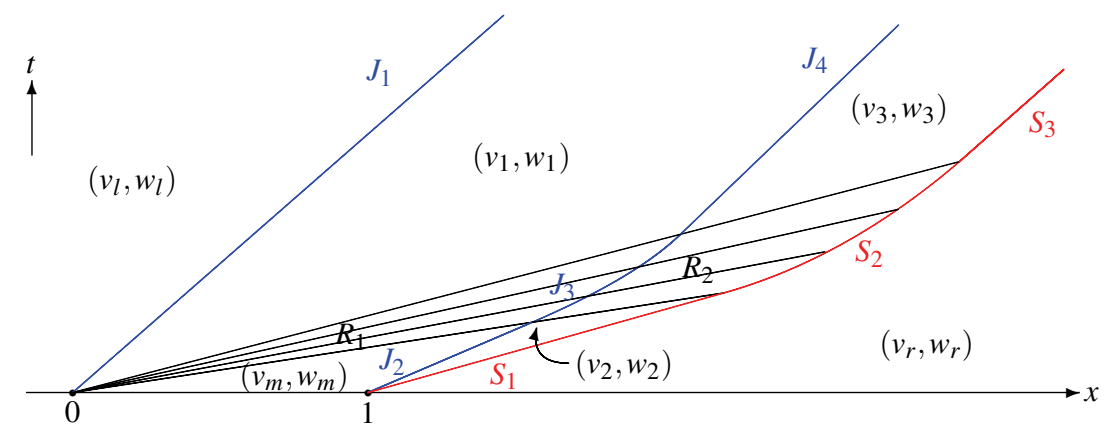

Fig.5.4. The global solution of the Cauchy problem (1.1) and (3.1) when $-1<v_{m}<v_{l}<v_{r}<0$.

Case 5.6. $-1<v_{m}<v_{r}<v_{l}<0$

In this case, we need also to study the interaction of $J+R$ and $J+S$ when the condition $-1<$ $v_{m}<v_{r}<v_{l}<0$ is satisfied. The situation in this case is similar to that in Case 5.5 and the difference only lies in that the shock wave cannot penetrate $R_{2}$ completely and finally has the characteristic line $x=\frac{t}{\left(1+v_{r}\right)^{2}}$ in $R_{2}$ as its asymptote.

In the end, we can also summarize our results for the zero rarefaction wave in the limit $v_{m} \rightarrow$ $(-1)^{+}$situation in the following theorem.

Theorem 5.1. If the initial condition (3.1) satisfies $-1<v_{m}<0, v_{m}<v_{l}$ and $v_{m}<v_{r}$, then a zero rarefaction wave generates at the point $(0,0)$ and keeps only in the interval $[0,1]$ in the positive $x$-axis in the limit $v_{m} \rightarrow(-1)^{+}$situation for the Cauchy problem (1.1) and (3.1).

\section{References}

[1] L. Ambrosio, G. Crippa, A. Figalli and L.A. Spinolo, Some new well-posedness results for continuity and transport equations, and applications to the chromatography system, SIAM J.Math.Anal. 41 (2009) 1890-1920.

[2] D. Armbruster, S. Gottlich and M. Herty, A scalar conservation law with discontinuous flux for supply chains with finite buffers, SIAM J. Appl. Math. 71 (2011) 1070-1087.

[3] A. Bressan, Hyperbolic Systems of Conservation Laws: The One-dimensional Cauchy Problem, Oxford Lecture Ser. Math. Appl. 20 (Oxford University Press, Oxford, 2000).

[4] A. Bressan and W. Shen, Uniqueness of discontinuous ODE and conservation laws, Nonlinear Anal. TMA 34 (1998) 637-652.

[5] G.Q. Chen and H. Liu, Formation of $\delta$-shocks and vacuum states in the vanishing pressure limit of solutions to the Euler equations for isentropic fluids, SIAM J. Math. Anal. 34 (2003) 925-938.

[6] H. Cheng and H. Yang, Delta shock waves in chromatography equations, J. Math. Anal. Appl. 380 (2011) 475-485.

[7] V.G. Danilov and D. Mitrovic, Delta shock wave formation in the case of triangular hyperbolic system of conservation laws, J. Differential Equations 245 (2008) 3704-3734.

[8] V.G. Danilov and V.M. Shelkovich, Dynamics of propagation and interaction of $\delta$-shock waves in conservation law systems. J. Differential Equations 221 (2005) 333-381.

[9] V.G. Danilov and V.M. Shelkovich, Delta-shock waves type solution of hyperbolic systems of conservation laws, $Q$. Appl. Math. 63 (2005) 401-427.

[10] J.P. Dias and M. Figueira, On the approximation of the solutions of the Riemann problem for a discontinuous conservation law, Bull. Braz. Math. Soc. 36 (2005) 115-125.

[11] T. Gimse, Conservation laws with discontinuous flux functions, SIAM J. Math. Anal. 24 (1993) 279 289. 
[12] S. Gottlich, A. Klar and P. Schindler, Discontinuous conservation laws for production networks with finite buffers, SIAM J. Appl. Math. 73 (2013) 1117-1138.

[13] L. Guo, L. Pan and G. Yin, The perturbed Riemann problem and delta contact discontinuity in chromatography equations, Nonlinear Analysis, TMA 106 (2014) 110-123.

[14] L. Guo, Y. Zhang and G. Yin, Interactions of delta shock waves for the Chaplygin gas dynamic equations with split delta functions, J. Math. Anal. Appl. 410 (2014) 190-201.

[15] F. Huang and Z. Wang, Well-posedness for pressureless flow, Comm. Math. Phys. 222 (2001) 117-146.

[16] H. Kalisch and D. Mitrovic, Singular solutions of a fully nonlinear $2 \times 2$ system of conservation laws, Proceedings of the Edinburgh Mathematical Society 55 (2012) 711-729.

[17] H. Kalisch and D. Mitrovic, Singular solutions for the shallow-water equations, IMA J. Appl. Math. 77 (2012) 340-350.

[18] B.L. Keyfitz and H.C. Kranzer, Spaces of weighted measures for conservation laws with singular shock solutions, J. Differential Equations 118 (1995) 420-451.

[19] J. Li, T. Zhang and S. Yang, The Two-Dimensional Riemann Problem in Gas Dynamics, Pitman Monographs and Surveys in Pure and Applied Mathematics, 98 (Longman Scientific and Technical, 1998).

[20] X. Li and C. Shen, Viscous regularization of delta shock wave for a simplified chromatography system, Abstract and Applied Analysis, Volume 2013, Article ID 893465, 10 pages.

[21] M. Mazzotti, Non-classical composition fronts in nonlinear chromatography - Delta-shock, Ind. Eng. Chem. Res. 48 (2009) 7733-7752.

[22] M. Mazzotti, A. Tarafder, J. Cornel, F. Gritti and G.Guiochon, Experimental evidence of a delta-shock in nonlinear chromatography, J. Chromatography. A 1217 (2010) 2002-2012.

[23] M. Nedeljkov, Delta and singular delta locus for one dimensional systems of conservation laws, Math. Methods Appl.Sci. 27 (2004) 931-955.

[24] M. Nedeljkov, Singular shock waves in interactions, Q. Appl. Math. 66 (2008) 281-302.

[25] M. Nedeljkov, Shadow waves: entropies and interactions for delta and singular shocks, Arch. Rational Mech. Anal. 197 (2010) 487-537.

[26] M. Nedeljkov, Higher order shadow waves and delta shock blow up in the Chaplygin gas, J. Differential Equations 256 (2014) 3859-3887.

[27] M. Nedeljkov and M. Oberguggenberger, Interactions of delta shock waves in a strictly hyperbolic system of conservation laws, J. Math. Anal. Appl. 344 (2008) 1143-1157.

[28] D.N. Ostrov, Asymptotic behavior of two interreacting chemicals in a chromatography reactor, SIAM J. Math. Anal. 27 (1996) 1559-1596.

[29] E.Yu. Panov and V.M. Shelkovich, $\delta^{\prime}$-shock waves as a new type of solutions to system of conservation laws, J. Differential Equations 228 (2006) 49-86.

[30] A. Qu and and Z. Wang, Stability of the Riemann solutions for a Chaplygin gas, J. Math. Anal. Appl. 409 (2014) 347-361.

[31] H.K. Rhee, R. Aris and N.R. Amundson, First-order partial differential equations, volume 2: theory and application of hyperbolic systems of quasilinear equations, (New York: Dover Publications, 2001).

[32] C. Shen, Wave interactions and stability of the Riemann solutions for the chromatography equations, $J$. Math. Anal. Appl. 365 (2010) 609-618.

[33] C. Shen and M. Sun, Formation of delta shocks and vacuum states in the vanishing pressure limit of Riemann solutions to the perturbed Aw-Rascle model, J. Differential Equations 249 (2010) 3024-3051.

[34] C. Shen and M. Sun, Interactions of delta shock waves for the transport equations with split delta functions, J. Math. Anal. Appl. 351 (2009) 747-755.

[35] C. Shen and M. Sun, Stability of the Riemann solutions for a nonstrictly hyperbolic system of conservation laws, Nonlinear Analysis, TMA 73 (2010) 3284-3294.

[36] W. Sheng and T. Zhang, The Riemann problem for the transportation equations in gas dynamics, Mem. Amer. Math. Soc. 137(N654) (1999), AMS:Providence.

[37] M. Sun, Delta shock waves for the chromatography equations as self-similar viscosity limits, $Q$. Appl. Math. 69 (2011) 425-443.

[38] M. Sun, Interactions of delta shock waves for the chromatography equations, Applied Mathematics Letters 26 (2013) 631-637. 
[39] D. Tan, T. Zhang and Y. Zheng, Delta-shock waves as limits of vanishing viscosity for hyperbolic systems of conservation laws, J. Differential Equations 112 (1994) 1-32.

[40] B. Temple, Systems of conservation laws with invariant submanifolds, Trans. Amer. Math. Soc. 280 (1983) 781-795.

[41] G. Wang, One-dimensional non-linear chromatography system and $\delta$-shock waves, Z. Angew. Math. Phys. 64 (2013) 1451-1469.

[42] J.K. Wiens, J.M. Stockiea and J.F. Williams, Riemann solver for a kinematic wave traffic model with discontinuous flux, J. Comp. Phys. 242 (2013) 1-23.

[43] H. Yang and Y. Zhang, New developments of delta shock waves and its applications in systems of conservation laws, J. Differential Equations 252 (2012) 5951-5993. 